\title{
REALIZACIÓN DE UN GRAFO EN RECINTOS RECTANGULARES SOBRE UNA PLANTA DEFINIDA
}

\author{
(MAPPING A RECTANGLE INTO RECTANGLES COVERING A GIVEN PLAN)
}

A. Recuero ${ }^{(1)}$, M. Alvarez ${ }^{(2)}$ y O. Río(1)

(1) IETCC/CSIC, Madrid; (2) Facultad de Informática-UPM, Madrid

ESPAÑA

Fecha de recepción: $26-\mathrm{V}-95$

403-17

\begin{abstract}
RESUMEN
En este trabajo se analiza el problema de la realización de un grafo en un conjunto de recintos rectangulares que cubran una planta dada, de lados paralelos a dos direcciones perpendiculares. Los grafos pueden representar el esquema básico de una vivienda o de un edificio y en él se muestran distintos requerimientos del proyecto. Para ello, se analiza el problema de la partición de un rectángulo en rectángulos, esto es, dado un grafo, saber si admite una realización de esta clase y, en caso afirmativo, generar automáticamente dichas realizaciones.
\end{abstract}

En ambos aspectos, se propone un procedimiento completo y general. Se trata de un problema de aplicación práctica en áreas tales como proyecto arquitectónico o compactación de circuitos electrónicos, del que no se ha encontrado una solución general en la bibliografia consultada.

\section{$S U M M A R Y$}

In this paper, the problem of mapping a graph into a set of rectangles covering a given plan is analized. The plan must have its sides parallel to two perpendicular directions. The graph may represent the basic scheme of a housing or a building, in which the different design requirements are collected. To this aim, the problem of partitioning a rectangle into rectangles is analysed.

It consists in determining whether a given graph can be realised this way, and if so, automatically generate some of its realizations. A general method is proposed to solve both aspects of the problem. It is a problem with practical application in areas such as architectural design or compaction of electronic circuits, to which no general solution has been found in the spetialized bibliography.

\section{Introducción}

El proyecto de un edificio es el resultado del proceso que realiza un arquitecto para la concreción espacial y formal de un programa de requerimientos previamente establecido. Tiene su plasmación en diferentes documentos (esquema básico, anteproyecto y proyecto). Para llegar a una sistematización del espacio arquitectónico, éste debe dividirse en células, normalmente rectangulares, del tamaño que interese, de manera que tapicen un soporte, normalmente también rectangular, o que puede transformarse en un rectángulo.

Se trata de crear una geometría en función de los requerimientos previamente establecidos y de un conocimiento profundo de la naturaleza del edificio. Para ello, se establecerán unas relaciones jerárquicas que determinen la importancia de cada célula o rectángulo y 
sus relaciones parciales y globales. De esta manera se crea la estructura funcional del edificio, que definirá la organización de usos y nexos de interrelación y la estructura formal de espacios para esos usos.

Tomando como base el programa, se generan espacios arquitectónicos, se organizan funcionalmente y formalmente con sus respectivos vínculos o nexos, dando lugar a una topología, representada por el esquema básico, cuya realización será la geometría deseada.

El esquema básico plasma las primeras ideas con sus variantes y alternativas, que una vez evaluadas y verificadas de acuerdo a pautas fijadas, darán lugar en una fase posterior al anteproyecto arquitectónico, donde se dan dimensiones a los esquemas, se define la conformación espacial con sus límites y el tipo de espacios, para concluir en una última fase en el proyecto arquitectónico.

La teoría de grafos es una de las técnicas más adecuadas para llevar a cabo el proyecto, al emplear relaciones de vecindad (relacionado con) y de conexión, basadas en los requerimientos de un sistema espacial. Es en las fases iniciales del proceso de proyecto, la elaboración de esquemas básicos, donde el trabajo que se describe puede ser de ayuda para la obtención de alternativas que respondan a unos requerimientos funcionales previamente establecidos en el programa arquitectónico. Los esquemas básicos son plasmados en grafos que definen los vínculos funcionales, precisando el tipo y grado de relación existente entre las diferentes células o rectángulos, representando los vértices del grafo el conjunto finito de las mismas y los arcos sus relaciones. De estos análisis puede deducirse que dos vértices han de estar necesariamente conectados o desconectados, oque puede admitirse queestén conectados. El conjunto de todos los grafos que reflejan las conexiones admisibles se obtendrá agregando al grafo que recoge las conexiones obligatorias, las distintas combinaciones de las que pueden existir o no.

En este trabajo, se consideran las realizaciones de un grafo concreto, esto es, en el que los rectángulos de su realización estarán en contacto si, y sólo si, existe un arco entre los vértices que los representan. A partir del grafo primario se puede inferir un grafo secundario, o esqueleto de la partición, en el cual los arcos se convierten en fronteras de las células o rectángulos. Este esqueleto representa topológicamente la estructura intercelular del sistema espacial, de la cual se puede inferir la geometrización del proyecto arquitectónico.

El que un grafo admita numerosas realizaciones significa que, unavez que el proyectista ha descrito sus requerimientos en forma de grafo, existe una gran variedad de soluciones arquitectónicas posibles. La ventaja de realizar algunas de las operaciones necesarias a nivel del grafo abstracto, en lugar de hacerlo sobre planos, es que las decisiones pueden ser objetivas, sin tener en cuenta consideraciones relativas a la forma $o$ aspecto de las habitaciones.

El problema de saber si un grafo es realizable en recintos rectangulares que cubran una planta dada de forma cualquiera, de lados paralelos a dos direcciones perpendiculares, no parece abordable de forma directa. La vía elegida por los autores ha sido la de considerar, en primer lugar, el caso de planta rectangular. Resuelto el problema en dicho caso, se propone una forma de reducir el caso general al caso resuelto, mediante la introducción de grafos auxiliares, que complican el grafo de partida pero que permiten pasar de un problema no resuelto a otro resuelto.

Este trabajo se ha realizado como parte del proyecto de investigación "Desarrollo, mejora e integración de sistemas $\mathrm{CAD} / \mathrm{CAE}$ en construcción: Aplicación al caso de puentes pretensados", financiado por la DGICYT, PB 93/0111, en el Instituto de Ciencias de la Construcción Eduardo Torroja, del CSIC.

Si bien se presenta aqui un algoritmo, no se ha utilizado un estilo matemático en su descripción, esto es, no se ha organizado en definiciones, teoremas, demostraciones, corolarios y lemas. De hecho, se propone un conjunto de condiciones necesarias que cubren todos los casos que los autores han podido imaginar, pero no se demuestra que su cumplimiento sea condición suficiente. Se ha tratado de hacer una presentación intuitiva, explicando los conceptos no habituales en la primera ocasión que aparecen.

\section{Partición de un rectángulo en rectángulos}

Considérese un rectángulo dividido en un conjunto de rectángulos. Si situamos en cada rectángulo un vértice y unimos mediante un arco los vértices correspondientes a aquellos que tengan algún trozo de lado común, obtendremos un grafo asociado a la partición, tal como se muestra en la figura 1. Recíprocamente, si un grafo puede estar asociado a una partición del tipo descrito, diremos que es realizable, y que la partición es una realización del grafo. Esta es una acepción restringida del concepto, pues en general no se impone la condición de que cubran un rectángulo. Una partición da lugar a un solo grafo, mientras que un grafo realizable puede dar lugar a numerosas realizaciones. En la figura 2 se muestran tres realizaciones del grafo de la figura 1B distintas de la mostrada en la $1 \mathrm{~A}$.

En dicho trabajo se aborda el problema de determinar si un grafo es realizable y, en caso afirmativo, generar automáticamente realizaciones del mismo. Para ello, se analizan las peculiares características geométricas que impone el usar sólo rectángulos para tappizar el rectángulo total y como se traducen éstas en condiciones topológicas que debe cumplir un grafo para ser realizable. 


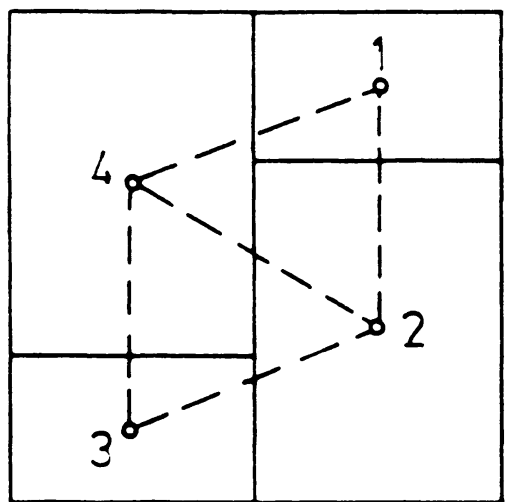

A

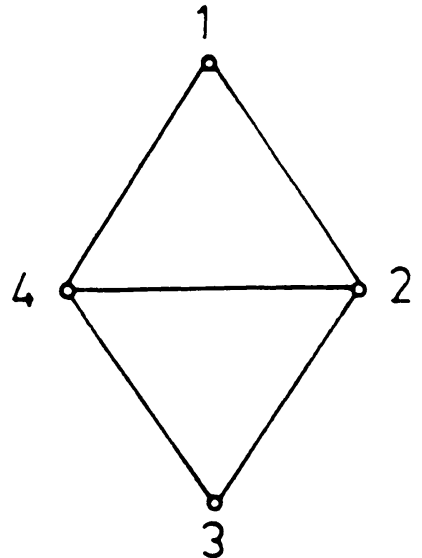

B

Fig. 1

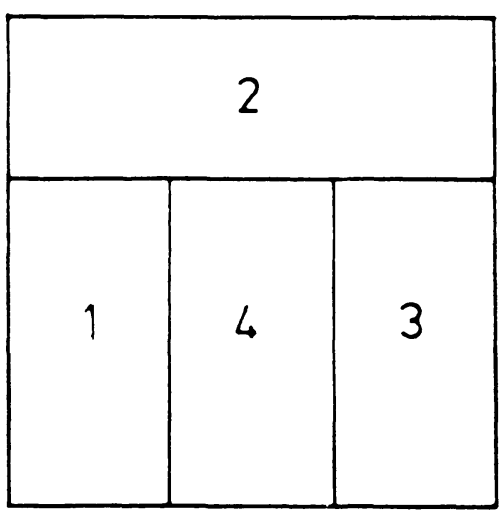

A

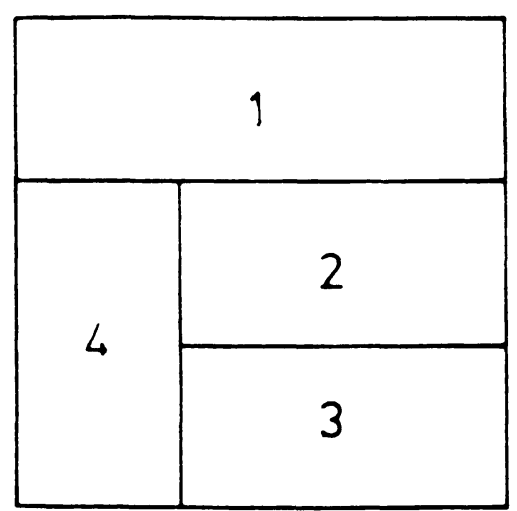

B

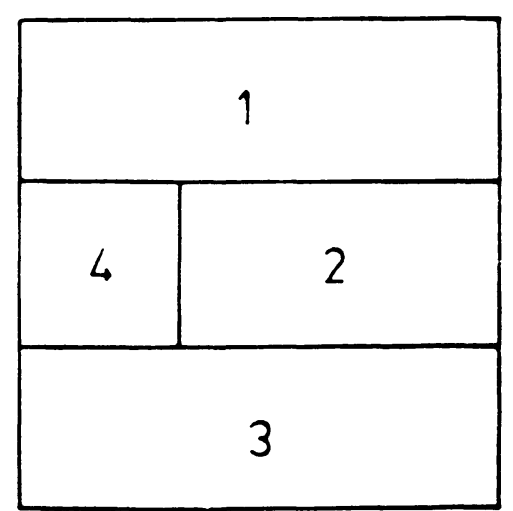

C

Fig. 2

Este problema tiene aplicación práctica en campos tales como el proyecto automatizado de distribuciones arquitectónicas o el de compactación de circuitos electrónicos integrados. Fue planteado por Ungar en 1953, $\mathrm{y}$, aunque ha sido abordado, más o menos directamente, por diversos autores Levin, Mitchel et al., Steadman, Earl, March, Magiera, Roth et al., Schwarz et al., entre otros, los autores no han encontrado ninguna referencia en la que se presente una solución completa del mismo.

Magiera presenta algunas propiedades de las particiones exteriores de un rectángulo en rectángulos y estudia los grafos asociados con ellas. Tan solo considera rectángulos exteriores, esto es, aquéllos que tienen al menos un lado sobre el contorno exterior.

En los modelos propuestos por Roth et al. y por Schwarz et al. no se trata de cubrir una planta prefijada. El primero utiliza grafos que representan las paredes en ambas direcciones y aplica técnicas de tipo PERT para obtener planos con dimensiones optimizadas de las habitaciones. El segundo utiliza dos digrafos ponderados, cada uno de ellos representando las paredes en una dirección y aplica técnicas utilizadas en la compactación en dos dimensiones de circuitos VLSI (Very Large Scale Integration)

En estosúltimos métodos se utilizan técnicas desarrolladas en otras áreas y es necesario tomar decisiones previas sobre posiciones relativas entre los rectángulos. Sin embargo, hay requerimientos que pueden cumplirse de varias formas, tal como que dos habitaciones puedan ser contiguas en cualquier dirección. El método propuesto aquí, es más general, pues no impone condiciones a priori sobre las posiciones relativas entre habitaciones, si bien, en principio, es sólo válido para plantas rectangulares, que son las únicas convexas que pueden ser cubiertas por rectángulos. Sin 
embargo, se verá como puede aplicarse a plantas no convexas, siempre que sus lados sean paralelos a dos direcciones perpendiculares. Las condiciones sobre dimensiones, tales como valores máximos o mínimos de longitudes o superficies, pueden ser introducidas en distintos puntos del algoritmo.

\section{Realizabilidad de un grafo}

Para estudiar la realizabilidad de un grafo, se introduce el concepto de envoltura de un rectángulo. Considérese un rectángulo cualquiera, al que llamaremos rectángulo base,

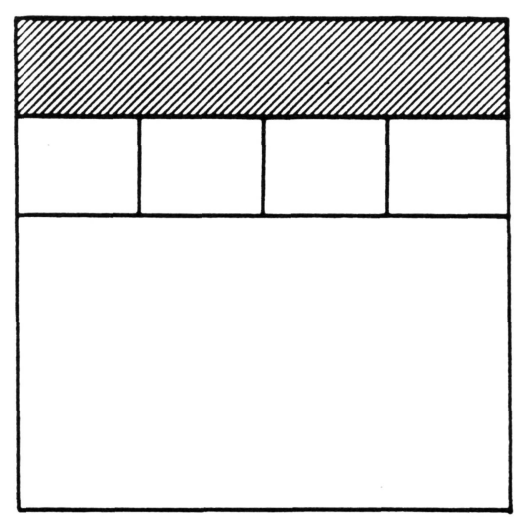

A

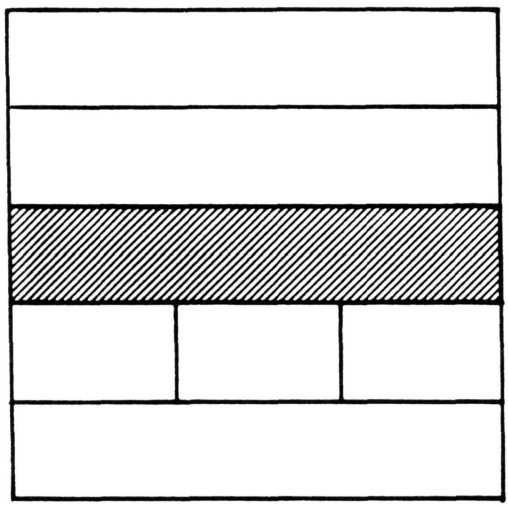

C tal como el sombreado en las distintas realizaciones de la figura 3; se denominará envoltura del mismo al conjunto de rectángulos que tienen algún contacto con él, ya sea en parte de un lado o en un vértice.

Dos rectángulos que sólo están conectados por un vértice se dice que son verticales entre sí. Si existiesen dos rectángulos verticales entre sí, habrá otra pareja de rectángulos verticales entre sí en el mismo vértice. En consecuencia, los rectángulos verticales entre sí siempre se producen en grupos de 4 , verticales dos a dos.

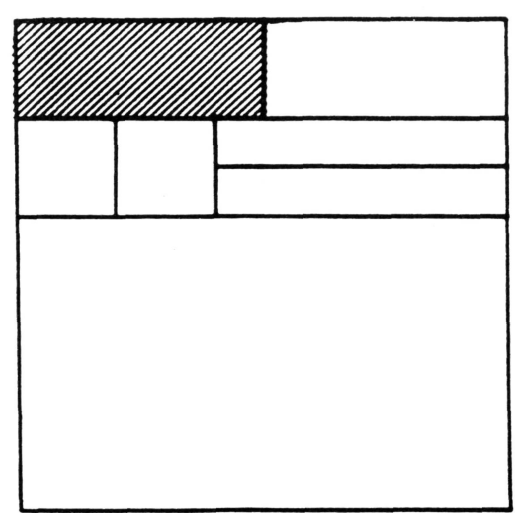

B

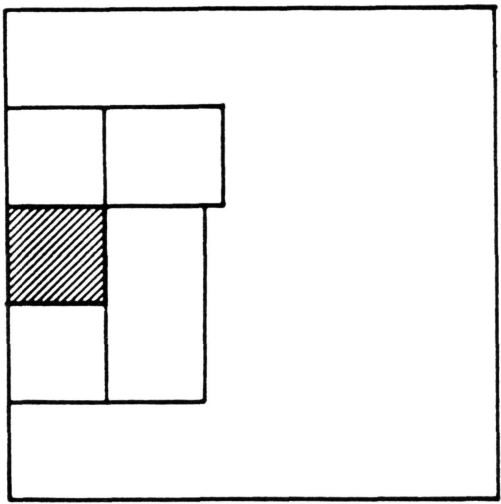

D

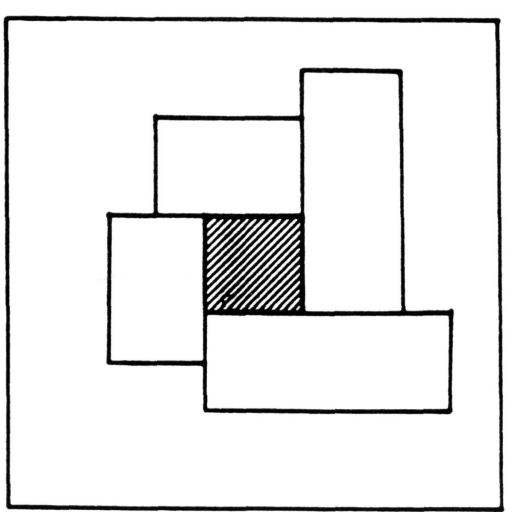

E 
Si los vértices de la envoltura forman un ciclo se dirá que es cerraday, en caso contrario, que es abierta. Un rectángulo interior tendrá una envoltura cerrada, en tanto que uno exterior, es decir con algún lado sobre el contorno exterior, tendrá una envoltura abierta.

En los 5 casos de la figura 3, la envoltura está formada por 4 rectángulos. Laenvoltura puede tener una parte (casos A, $B, D, E)$ o dos partes independientes (caso C), según que todos dichos rectángulos estén situados de forma contigua o no. La envoltura tendrá dos partes cuando el rectángulo base tenga un lado que ocupe una dimensión completa del rectángulo total y tenga rectángulos a ambos lados (caso $\mathrm{C}$, pero no caso A). Si la envoltura es continua, puede cu-brir totalmente cualquier número de lados consecutivos del rectángulo base, según cual sea su posición ( 1 en A, 2 en $B, 3$ en D y 4 en E). Veamos algunas condiciones que cumplen necesariamente los rectángulos de la envoltura.

- Los rectángulos de cada parte de la envoltura han de formar una sucesión ordenada, es decir, cada rectángulo puede tener uno anterior y uno posterior.

- Si la envoltura tiene una sola parte, ésta puede ser cerrada, formando, en tal caso, sus rectángulos un ciclo con 4 elementos más el número de rectángulos verticales al base, como mínimo. En la figura 4 se muestran ejemplos de los 5 casos posibles de envoltura cerrada formada por el mínimo número de rectángulos, según el número de rectángulos verticales al base.

- Si tuviese más de dos rectángulos verticales al base, la envoltura deberá ser cerrada.

- Si laenvoltura tuviese dos partes, una de las dimensiones del rectángulo base ocupará una dimensión completa del rectángulo total y no podrán existir rectángulos verticales al base en la envoltura.

- Si una parte de la envoltura incluye un rectángulo que exige un lado completo, éste será el único rectángulo de dicha parte.

- Si la envoltura tuviese dos partes, los rectángulos situados a uno y otro lado del base no pueden tener contacto. En consecuencia, si se suprimiese el vértice base, junto con todas las aristas que concurren en él, el grafo quedaría dividido en dos subgrafos separados.

- Si un rectángulo ocupase un extremo completo, esto es, un lado completo del rectángulo total, y estuviese envuelto por un solo rectángulo, su vértice asociado en el grafo sería de grado 1 . Esta es la única realización que admite un vértice de grado 1 , por lo que no podrán existir más de dos vértices de este tipo en el grafo. En la figura 5 puede verse una partición con dos rectángulos extremos, y su correspondiente grafo, uno realización de un vértice de grado 1 yel otro no. Esteúltimoadmite otras realizaciones, pero no así el primero.

- El rectángulo adyacente a uno realización de un vértice de grado 1, ha de ocupar una dimensión completa del rectángulo total.

- No pueden existir más que grupos de cuatro rectángulos coincidentes en un punto.

Veamos cómo puede construirse la envoltura de un rectángulo base cualquiera a partir de la descripción del grafo. Para ello, se utilizará como ejemplo el grafo de la figura 6, en el que se estudiará la envoltura del vértice 1.

- Formarán parte de ella todos los vértices adyacentes al de base. En el ejemplo, los vértices 2, 3, 4, 6, 8 y 9 .

- Comprobar que pueden agruparse en conjuntos de vértices consecutivos, cada uno de ellos con 1 o más vértices unidos a lo sumo a otros dos vértices del mismo conjunto; en caso contrario el grafo no sería realizable. Tampoco lo sería si hubiese más de 4 de dichos conjuntos. En el ejemplo, hay 3 grupos, formados por los vértices 2,3 y 4 el primero, por el 6 el segundo y por los 8 y 9 el tercero.

- Si hubiese un solo conjunto y todos sus vértices estuviesen conectados a otros dos, formarían un ciclo, que debería ser de longitud 4 o mayor para que el grafo pudiese ser realizable.

Para detectar la existencia de rectángulos verticales al de base, se comprobará si existen vértices adyacentes simultáneamente (distintos del de base) a los vértices extremos de dos de dichos conjuntos. Los vértices extremos estarán conectados a uno o ningún otro vértice del mismo conjunto, según que el mismo conste de dos o más vértices o sólo de uno. En el ejemplo, los extremos son los vértices 2 y 4 , el 6 y los 8 y 9 ; los verticales al 1 son el 5 , que conecta el 4 y el 6 , y el 7 que conecta el 6 y el 8 .

- Si existiese un vértice adyacente común, distinto del de base, a los extremos de un conjunto de más de tres vértices, y éste fuese el único conjunto, dicho vértice podrá ser vertical al de base o no serlo, con lo que la envoltura podrá ser abierta o cerrada, condición a determinar por otros criterios. Considérese el caso del grafo de la figura $7 \mathrm{~A}$, en el que todos sus vértices son equivalentes. Cada vértice está conectado a otros 4 y los extremos del grupo tienen otro vértice adyacente común. En la realización del grafo de la figura 7B, cuatro vértices tienen una envoltura cerraday los otros cuatro la tienen abierta. Si se diese esta circunstancia, pero el grupo no fuese único, el vértice no será vertical al de base.

- Si un vértice vertical conectase dos conjuntos distintos, éstos se unirían. En cualquier caso, se incorporará el vértice vertical al conjunto, único o resultante de la unión. 


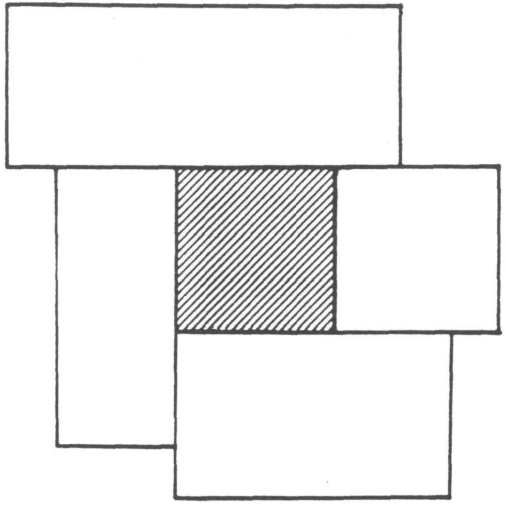

A

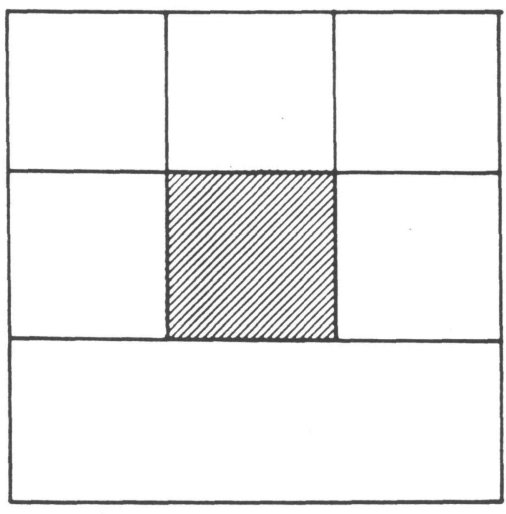

C

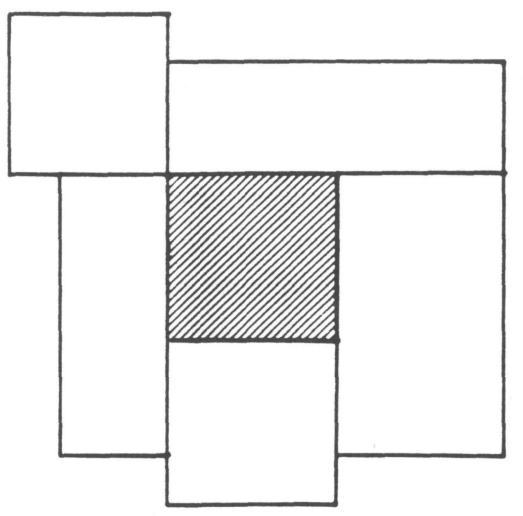

B

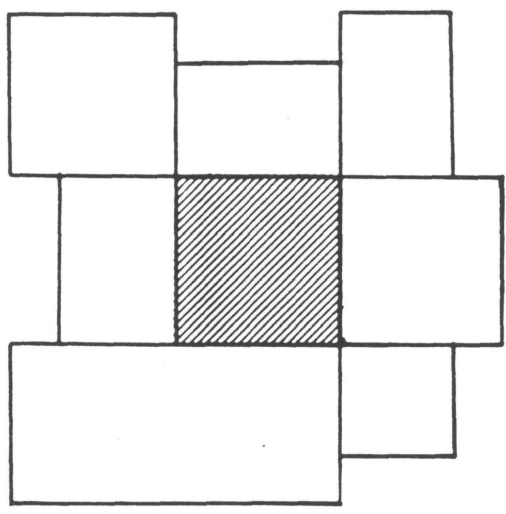

D

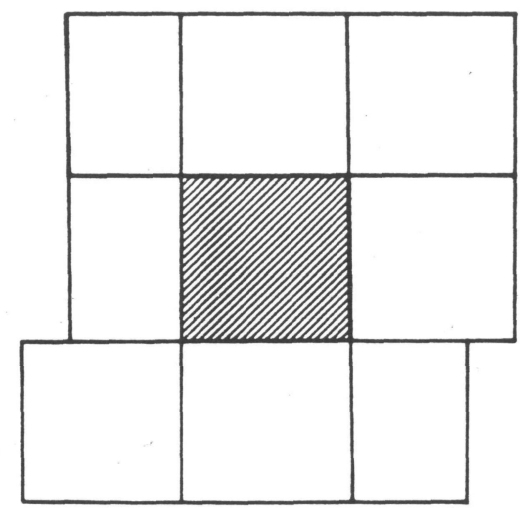

$E$

Fig. 4

(c) Consejo Superior de Investigaciones Científicas Licencia Creative Commons 3.0 España (by-nc) 


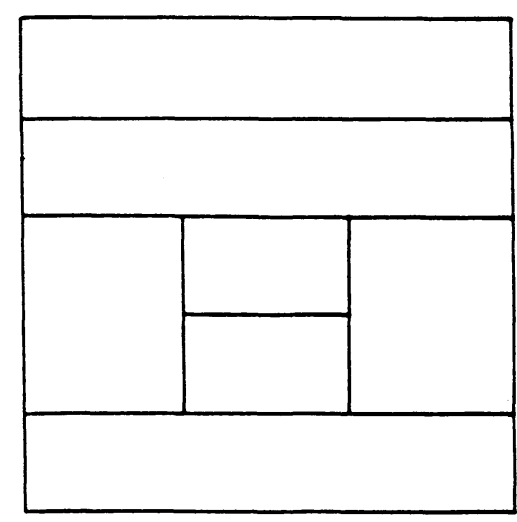

A

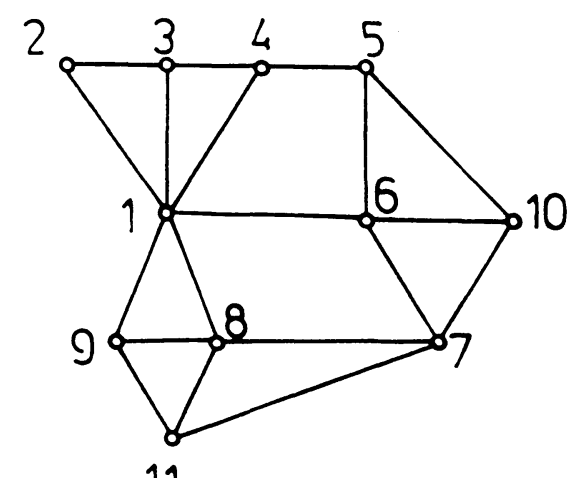

11

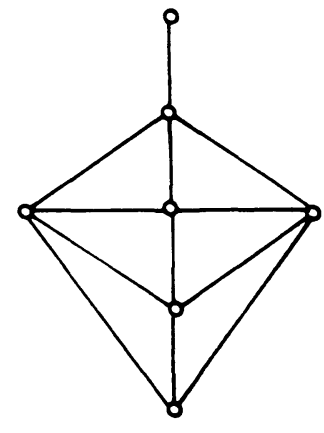

Fig. 5

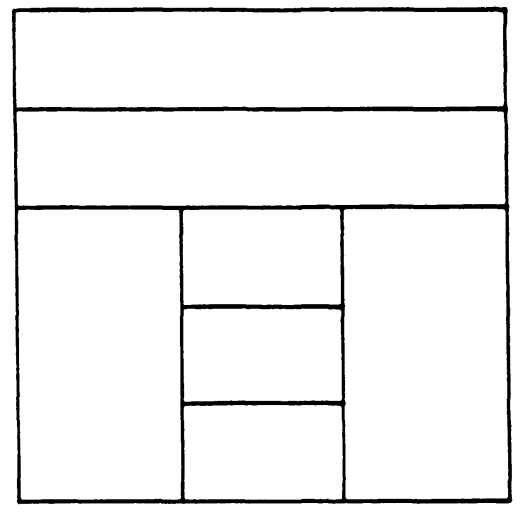

B

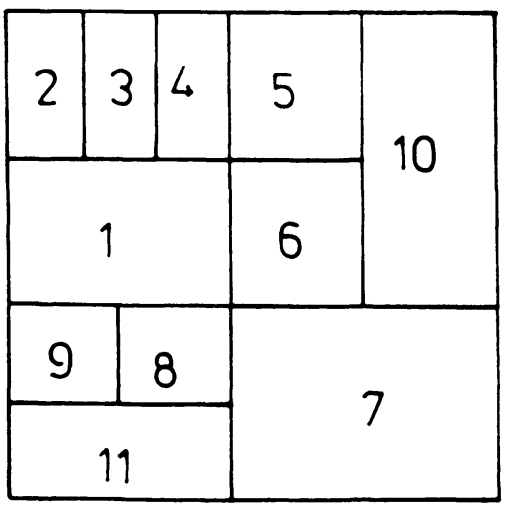

Fig. 6

Al final de este proceso puede quedar un solo conjunto abierto o cerrado, formado por vértices adyacentes al de base y por vértices verticales al mismo, o un solo conjunto indefinido, formado por vértices adyacentes al de base $y$ un vértice (que puede ser vertical al de base o no serlo), 0 dos conjuntos abiertos formados sólo por vértices adyacentes al de base, para que el grafo pueda ser realizable. Quedaría así completada la envoltura.

Si todos los vértices del grafo admiten una envoltura válida, deben hacerse comprobaciones globales sobre los mismos.
- Si el grafo tiene 4 vértices o menos, todos deben ser exteriores. Si tiene más de 4 vértices, al menos 4 de ellos han de ser exteriores. Así, en el caso de la figura 7A, para que el grafo sea realizable, al menos 4 de sus 8 vértices han de ser considerados exteriores. Además, puesto que los vértices verticales se producen en grupos de 4 , deberán ser exteriores o los 4 o los 8 vértices.

- Si todos los vértices del grafo fuesen exteriores, deberá haber entre ellos al menos dos de grado 1 ó 2 . En las realizaciones del grafo, los rectángulos asociados con estos vértices ocuparán necesariamente sendas esquinas del 
rectángulo total. En el grafo de la figura 7A, todos los vértices son de grado 4 , por lo que no pueden ser todos exteriores. Así, necesariamente, en dicho grafo 4 vértices deben ser exteriores y otros 4 interiores. La elección de uno de los vértices como interior obliga a considerar un grupo de 4 vértices verticales entre sí.

Algunas de las situaciones de indeterminación sobre si una envoltura es cerrada $o$ abierta pueden despejarse al hallar la envoltura de un vértice adyacente al dudoso. En otras ocasiones, serán las condiciones globales las que permitan la definición. Si persistiese la indefinición sobre la clase de envoltura de algún vértice, ésta podrá ser considerada alternativamente abierta o cerrada.

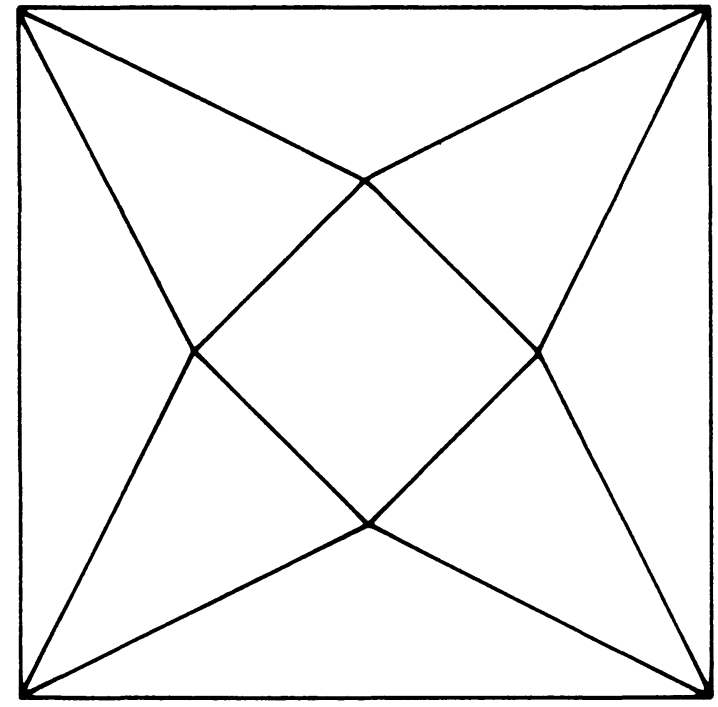

A

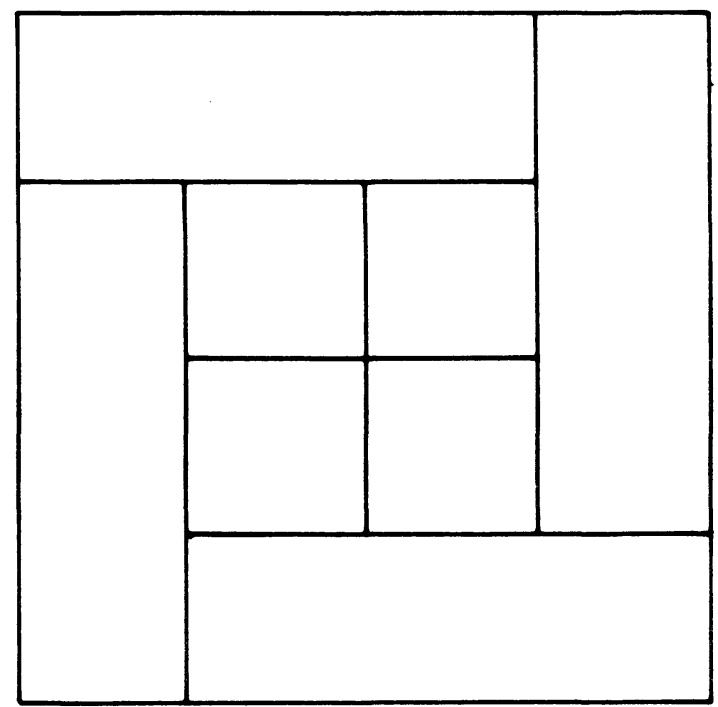

B

Fig. 7

\section{Algoritmo para la comprobación de la realizabilidad}

El algoritmo que se describe a continuación permite verificar el cumplimiento de las condiciones citadas en el apartado anterior. Realiza primero unas verificaciones globales, acerca de la conectividad y del grado de los vértices, seguidamente otras locales que deben cumplirse en cada uno de los vértices $\mathrm{y}$, por último, comprobaciones globales sobre el número de vértices exteriores. Si el grafo superase todas las verificaciones se considera que será realizable. El tiempo de ejecución (complejidad) del algoritmo es proporcional al cuadrado del número de vértices. En la tabla 1 se indican las operaciones a realizar y las verificaciones subsiguientes a cada operación.

\section{Ejemplos de grafos no realizables}

Se presentan aquí varios ejemplos de grafos no realizables, indicando cual es la razón de esta circunstancia y cual de las verificaciones del algoritmo la detectaría. En primer lugar, puesto que no pueden existir más de dos vértices de grado 1, no serán realizables los árboles que consten de más de una rama, esto es que tengan algún vértice de grado mayor que 2, como es el caso de la figura $8 \mathrm{~A}$. Por idéntica razón, no es realizable el grafo 8B. Ambos casos son detectados en la verificación V2.a. El grafo $8 \mathrm{C}$ no es realizable por haber más de 4 conjuntos de vértices adyacentes al de base, condición detectada por V3.2a. En el grafo 8D hay un vértice de grado local 3 en el conjunto de vértices adyacentes al de base, detectado en $\mathrm{V} 3.2 \mathrm{~b}$. El grafo completo de orden $4(\mathrm{~K} 4)$ no es realizable, figura $8 \mathrm{E}$, pues sus vértices están rodeados por ciclos de longitud 3 . Tampoco lo serán los grafos completos de orden mayor que 4 , pues no pasan la verificación $\mathrm{V} 3.2 \mathrm{~b}$. El grafo $8 \mathrm{~F}$ tiene dos conjuntos de vértices adyacentes al de base, uno de ellos formando un ciclo, detectado por V3.2d. En los grafos $8 \mathrm{G}$ y $8 \mathrm{H}$, en un vértice adyacente a otro de grado 1 , hay más de dos conjuntos independientes de vértices adyacentes al de base, detectado por V3.2e. En el grafo 8I, dos vértices adyacentes a otros de grado 1 son adyacentes al de base y entre sí, pero no únicos, lo cual detecta V3.2f.

Los ejemplos siguientes muestran casos en los que la no realizabilidad del grafo se detecta al considerar los rectángulos verticales respecto al de base. En el grafo 9A hay dos vértices simultáneamente adyacentes a la misma pareja de vértices extremos, lo que es detectado por V3.3a. En el grafo 9B hay un vértice vertical al de base en la envoltura de uno adyacente a otro de grado 1 , detectado en V3.3b. El grafo 9C, que tenía inicialmente 4 conjuntos de vértices adyacentes al de base, tienefinalmente 3 al fusionarse dos de ellos por vértices verticales al de base, lo que es detectado en V3.4a. En el grafo 9D, una de las dos partes de la envoltura contiene un vértice vertical al de base, detectado en V3.4b. El grafo 9E tiene una envoltura abierta con 3 vértices verticales al de base, detectable con V3.4c. $\mathrm{El}$ grafo $9 \mathrm{~F}$ tiene una envoltura cerrada con un número 
TABLA 1

\begin{tabular}{|c|c|c|}
\hline $\mathrm{N}^{\circ}$ & OPERACIONES & VERIFICACIONES \\
\hline 1 & Comprobar si el grafo es conexo & a) Un grafo no conexo no es realizable \\
\hline 2 & $\begin{array}{l}\text { Contar } n^{\circ} \text { de vértices de grado } 1 \text {, y marcar vértice } \\
\text { adyacente. }\end{array}$ & a) No hay más de 2 vértices de grado 1 \\
\hline 3 & Efectuar en cada vértice del grafo: & \\
\hline 3.1. & Considerar todos los vértices adyacentes al base. & \\
\hline 3.2 . & $\begin{array}{l}\text { Agruparlos en conjuntos conexos, calculando el grado } \\
\text { local de cada vértice en dichos conjuntos. }\end{array}$ & $\begin{array}{l}\text { a) No hay más de } 4 \text { de dichos conjuntos. } \\
\text { b) No hay vértices con grado local }>2 \text {. } \\
\text { c) Si todos los vértices del conjunto son de grado local } 2 \text {, } \\
\text { ciclo debe tener } 4 \text { o más vértices. } \\
\text { d) Si un conjunto es un ciclo no puede haber otros } \\
\text { conjuntos. } \\
\text { e) Si el vértice base fuese adyacente a otro de grado } 1 \text {, no } \\
\text { puede haber más de un conjunto además del que el } \\
\text { contiene el vértice de grado } 1 \text {. } \\
\text { f) En el caso e), cuando exista un } 2^{\circ} \text { conjunto, si en éste } \\
\text { hubiese un vértice adyacente a otro de grado } 1 \text {, deberá ser } \\
\text { el único del conjunto. }\end{array}$ \\
\hline 3.3 . & $\begin{array}{l}\text { Comprobar si existen vértices adyacentes a cada pareja de } \\
\text { vértices extremos de un mismo conjunto de más de } 2 \\
\text { vértices (posibles rectángulos verticales). Si existe uno } \\
\text { adyacente a los extremos de un mismo grupo y sólo hay un } \\
\text { grupo de más de } 2 \text { vértices, dicho vértice puede o no ser } \\
\text { vertical al base; pero si hubiese más de un grupo; o sólo } \\
\text { hubiese un grupo de menos de } 3 \text { vértices, no sería vertical } \\
\text { al base. }\end{array}$ & $\begin{array}{l}\text { a) No puede haber más de un gráfico adyacente a una } \\
\text { misma pareja. } \\
\text { b) Si el base es adyacente a otro de grado } 1 \text {, no pueden } \\
\text { existir vértices verticales al base. }\end{array}$ \\
\hline 3.3 .1 & $\begin{array}{l}\text { Cada vértice vertical aumenta en } 1 \text { el grado local de los } \\
\text { vértices que conecta, y su grado local es } 2 \text {. Si los vértices } \\
\text { conectados perteneciesen a conjuntos distintos, éstos se } \\
\text { unirían en } 1 \text { sólo. }\end{array}$ & \\
\hline 3.4 . & $\begin{array}{l}\text { Anotar el } \mathrm{n}^{\circ} \text { de partes de su envoltura y si ésta es abierta, } \\
\text { cerrada o indefinida y para cada una de sus partes el } \mathrm{n}^{\circ} \mathrm{de} \\
\text { vértices. Para cada vértice, su grado local, si son o no } \\
\text { verticales al base o si requieren una dimensión completa. }\end{array}$ & $\begin{array}{l}\text { a) La envoltura sólo puede tener } 1 \text { o } 2 \text { partes. } \\
\text { b) Si tiene } 2 \text { partes no puede tener rectángulos verticales al } \\
\text { base. } \\
\text { c) Si tuviese más de } 2 \text { rectángulos verticales al base, la } \\
\text { envoltura ha de ser cerrada. } \\
\text { d) Si la envoltura tiene una parte cíclica (todos los vértices } \\
\text { de grado local =2) es única y tiene al menos tantos } \\
\text { vértices como rectángulos verticales al base más } 4 \text {. } \\
\text { e) Si el vértice base exige una dimensión completa, cada } \\
\text { parte de su envoltura no contiene más de un vértice que } \\
\text { también lo exija. Si lo tuviese, sería el único vértice de } \\
\text { dicha parte. } \\
\text { f) Los vértices tienen un grado local < } 3 \text {. }\end{array}$ \\
\hline 3.5 . & $\begin{array}{l}\text { Si la envoltura tuviese una sola parte, continuar en } 3 \text { hasta } \\
\text { verificar todos los vértices. }\end{array}$ & \\
\hline 3.5.2. & $\begin{array}{l}\text { Si tuviese dos partes, anotar como pertenecientes a cada } \\
\text { parte los vértices conectados con vértices de la misma } \\
\text { mediante caminos que no pasen por el vértice base. } \\
\text { Volver a 3, hasta que se verifiquen todos los vértices. }\end{array}$ & a) Ningún vértice pertenece a ambas partes. \\
\hline 4. & Si todos los vértices admiten una envoltura válida. & $\begin{array}{l}\text { a) Si el número de vértices del grafo es menor que } 5 \text {, } \\
\text { todos ellos son exteriores y si es mayor, al menos } 4 \text { de } \\
\text { ellos lo son. } \\
\text { b) Si todos los vértices son exteriores, atmenos dos de } \\
\text { ellos son de grado } 1 \text { o } 2 \text {. }\end{array}$ \\
\hline
\end{tabular}




$$
\begin{aligned}
& A \Delta \mathbb{A} \\
& \hat{A} \Delta \mathbb{Z} \\
& \dot{H}
\end{aligned}
$$




$$
\begin{aligned}
& \square \bar{\square} \\
& \infty \bar{\Delta} \\
& \otimes \bar{\square} \\
& \otimes \\
& \otimes
\end{aligned}
$$


insuficiente de vértices, detectado por V3.4d. En el grafo $9 \mathrm{G}$, el vértice adyacente al de grado 1 tiene en la otra parte de su envoltura un vértice que exige una dimensión completa junto con otro vértice, lo que es detectado por V3.4e. En el grafo $9 \mathrm{H}$, al unir los conjuntos iniciales con vértices verticales al de base, hay vértices de grado mayor que 2 en la envoltura, detectado por V3.4f.

Veamos otros tipos de causa de no realizabilidad. En el grafo 9I, la envoltura de cada vértice tiene dos partes, pero al suprimirlo el grafo no queda dividido en dos partes inconexas. Esta circunstancia es detectada por V3.5. 1a. En el grafo 9J, (16 vértices, todos ellos con envoltura cerrada formada por 4 vértices adyacentes y 4 verticales) todos los vértices son interiores, lo que es detectado por V4a. En el grafo $9 \mathrm{~K}$, (10 vértices, todos ellos con envoltura abierta formada por 4 vértices adyacentes) todos sus vértices son exteriores y de grado 4, lo que es detectado por V4b.

Evidentemente un grafo no plano no es realizable. El teorema de Kuratowski, dice que la condición necesaria y suficiente para que un grafo no sea plano es que contenga alguno de los grafos de la figura 10 , que son los grafos $K 3,3$

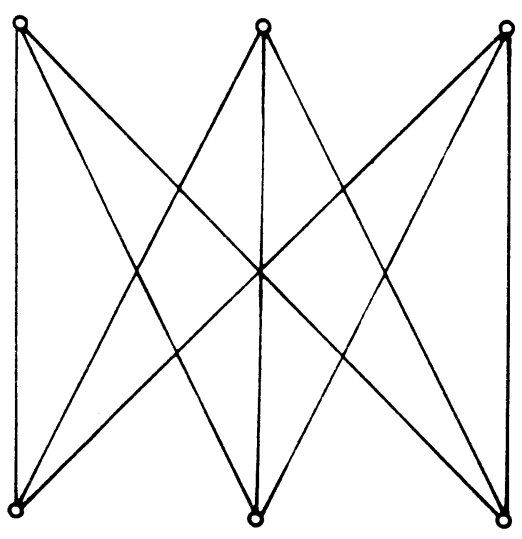

A

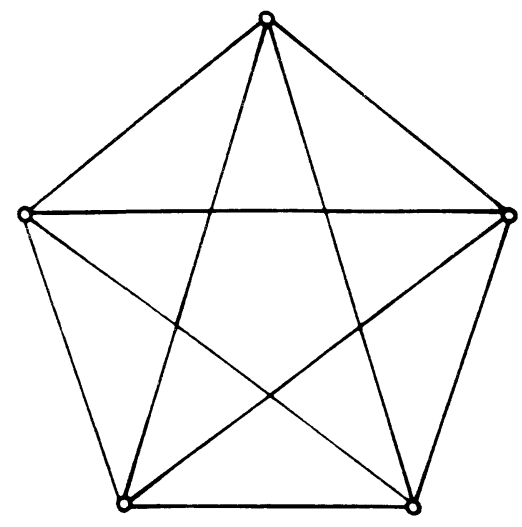

B

Fig. 10 (fig. 10A) y $\mathrm{K} 5$ (fig 10B) respectivamente. El algoritmo detecta la existencia de ambos. El caso de K3,3, o de cualquier grafo que lo contenga, puede ser detectado por V3.2d o por la V3.4f, dependiendo del orden en que se tomen los arcos del grafo. La verificación V3.2b detecta la no realizabilidad de $\mathrm{K} 5$ y la de cualquier grafo que lo contenga.

\section{Realizaciones del grafo}

Como yase havisto, si un grafoes realizable, pueden existir numerosas realizaciones del mismo. Hasta el momento, no se conoce el modo de saber cuantas realizaciones son posibles, ni la forma de generarlas automáticamente. $\mathrm{Pa}-$ ra abordar el problema, se tratará primero de simplificar los grafos en estudio, fragmentándolos en subgrafos que no incluyan rectángulos que hayan de cubrir necesariamente una dimensión completa del rectángulo total.

Si el grafo contiene rectángulos que exijan una dimensión completa, éstos pueden ser eliminados, imponiendo a sus rectángulos adyacentes la condición de cubrir entre todos una dimensión completa. De este modo, cualquier grafo realizable puede reducirse a uno o varios grafos, todos ellos con más de 3 vértices, también realizables, sin rectángulos que exijan una dimensión completa.

Los rectángulos pueden clasificarse en interiores y exteriores. Serán exteriores aquellos rectángulos en los que necesariamente, al menos uno de sus lados, forma parte del contorno exterior. Serán interiores aquéllos que han de estar necesariamente rodeados por otros rectángulos en sus cuatro lados. La envoltura de cada rectángulo permite establecer fácilmente esta división: serán exteriores los rectángulos con envoltura abierta e interiores los rectángulos con envoltura cerrada.

Los rectángulos exteriores pueden ordenarse formando una secuencia (ciclo hamiltoniano) de rectángulos consecutivos. Si alguno de ellos fuese de grado 2 , será necesariamente un rectángulo de esquina. Así, en el ejemplo de la figura 11 , son exteriores los rectángulos $1,2,3$, 8,9 y 10 . El rectángulo 8 es necesariamente de esquina, y los 1 y 9 han de ser adyacentes a él, cubriendo los otros dos lados del rectángulo 8. El orden en que deben estar los rectángulos exteriores es $1,8,9,10,3$ y 2 .Cualquier distribución que cubra los cuatro lados del rectángulo total, con el 8 en una esquina, será válida. La figura 12 muestra un conjunto de estas soluciones.

Los rectángulos interiores se irán situando sucesivamente. Se denominará capa interna al conjunto de rectángulos ya colocados que tengan todavía conexiones pendientes de ser satisfechas, esto es, con rectángulos sin colocar. Se considerará entonces el conjunto de rectángulos adyacentes a los de la capa interna, eliminando aquéllos que sean adyacentes a rectángulos no consecutivos de ésta. Se 

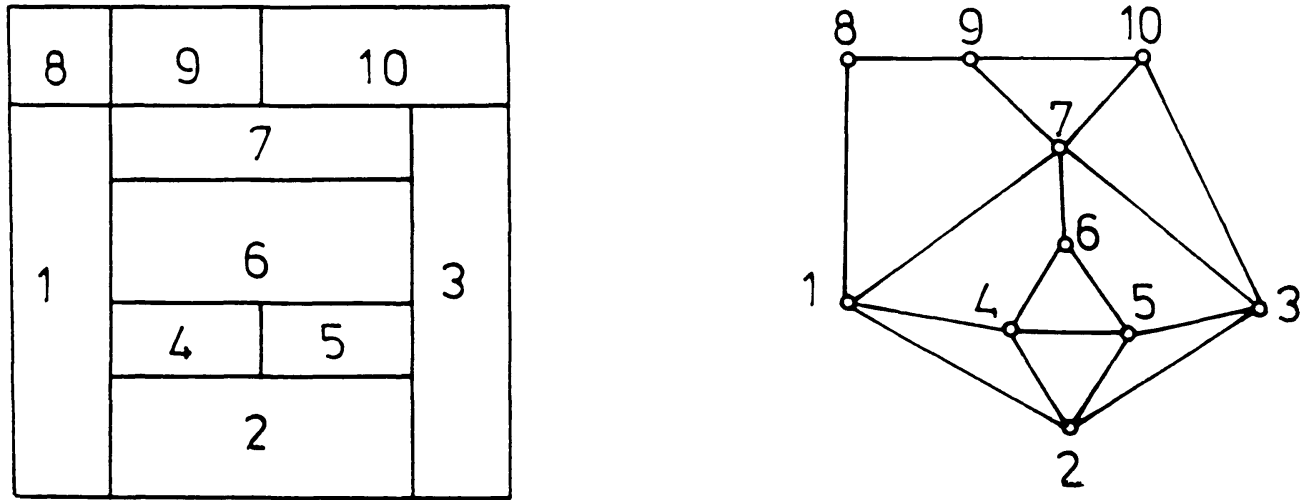

Fig. 11

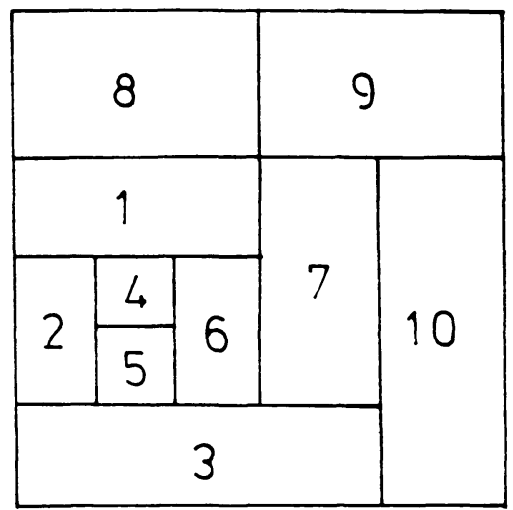

tomará como siguiente el que satisfaga mayor número de conexiones pendientes con la capa interna y se repetirá el proceso hasta colocar todos los rectángulos.

Todo esto permite determinar un orden de consideración de los vértices del grafo, que dependerá del orden en que se tomen los rectángulos, en los casos en que hubiese más de uno que satisficiese igual número de conexiones. En el ejemplo de la figura 11, la primera capa interna estará formada por los rectángulos $1,9,103,2$ y de nuevo el 1 . Los rectángulos adyacentes son los $4,5,6$ y 7 , pero se excluye el 6 por conectarse con rectángulos no consecutivos. Se tomará el 7 , que satura 3 conexiones. La nueva capa interna estará formada por $1,7,3$ y 2 . Se tomarán sucesivamente los rectángulos 6,4 y 5 .

El paso siguiente será el establecer una posición relativa válida entre los distintos rectángulos, para lo cual se hará uso del esqueleto de la partición.

\section{Esqueleto de una partición}

Se denomina esqueleto de una partición al grafo cuyos vértices son todos los vértices de los rectángulos y cuyas aristas son los segmentos de los lados entre cada dos vértices consecutivos. La propia partición es una representación del esqueleto. El número de vértices del esqueleto es: $\mathrm{NS}=2 * \mathrm{~N}+2-\mathrm{V}$, donde $\mathrm{N}>2$ es el número de vértices del grafo y $\mathrm{V}$ es el número de conjuntos de 4 rectángulos verticales dos a dos que existen. De ellos, $\mathrm{NSE}=\mathrm{NE}+4$, donde $\mathrm{NE}$ es el número de vértices exteriores del grafo, estarán en el contorno exterior. En consecuencia, el número de vértices del esqueleto interiores al rectángulo total será NSI $=2 * \mathrm{~N}-\mathrm{NE}-2-\mathrm{V}$. Así, en el grafo de la figura $1, \mathrm{~N}=4, \mathrm{NE}=4, \mathrm{~V}=0, \mathrm{NS}=10, \mathrm{NSE}=8$ y $\mathrm{NSI}=2$, y en el grafo de la figura $11, \mathrm{~N}=10, \mathrm{NE}=6, \mathrm{~V}=1, \mathrm{NS}=21, \mathrm{NSE}=10$ y NSI=11.

Para dibujar el esqueleto, de forma acorde con la partición, han de fijarse las coordenadas $x$ ey de cada vértice. De ellas, sólo $\mathrm{N}-1-\mathrm{V}$ son independientes, de las que NE-1-VE marcarán las posiciones de los vértices situados en el contorno exterior, exceptuados los 4 vértices de las esquinas, donde VE es el número de conjuntos de 4 cuadros verticales dos a dos que incluyen algún rectángulo exterior.

Denominaremos "grados de libertad de la partición"(GDL) al número de coordenadas independientes de su esqueleto. 
Sus valores podrán fijarse atendiendo a condiciones adicionales, tales como límites de superficie de los rectángulos, de dimensiones o relación entre ellas, etc.

Sean $\left.\right|_{x} i$ y $l_{y} i$ las dimensiones del rectángulo $i$ en las direcciones $\mathrm{x}$ e y respectivamente. El grafo de la figura 1 $(\mathrm{VE}=0)$ tiene $3 \mathrm{GDL}$, por ejemplo $\left.\right|_{x} 1,\left.I_{y} 1 \mathrm{y}\right|_{y} 2$, todos ellos ligados a vértices exteriores. El de la figura 11 tiene $8 \mathrm{GDL}$, 4 ligados a vértices exteriores, por ejemplo, $I_{x} 1,\left.\right|_{y} 1,|x 2 y|_{x} 9$, y 4 a interiores, por ejemplo, $\left.\right|_{y} 2,\left.\right|_{x} 4,\left.\right|_{y} 4$ y $\left.\right|_{y} 6$. Estos GDL pueden tomar valores positivos dentro de unos rangos definidos.

Si suponemos que las dimensiones del rectángulo total son la unidad, en los ejemplos citados tendremos que:

$$
\left.\right|_{x} 1<1,\left.\right|_{y} 1<\left.\right|_{y} 2<1 \text { en el grafo de la figura } 1,
$$

y que:

$$
\begin{aligned}
& \left.\right|_{x} 1+\left.\right|_{x} 2<1,\left.\right|_{y} 1<1, \\
& \left.\right|_{y} 2+\left.\right|_{y} 4+\left.\right|_{y} 6<\left.\right|_{y} 1, \\
& \left.\right|_{x} 1+\left.\right|_{x} 9<1, \\
& \left.\right|_{x} 4<\left.\right|_{x} 2 \text { en el grafo de la figura } 11 .
\end{aligned}
$$

Esta selección de los GDL no es única. El problema que se planteaes seleccionar los GDL del esqueleto de la realización elegida, expresar las coordenadas de todos sus vértices en función de ellos y establecer las ecuaciones o inecuaciones que resulten de imponer las condiciones topológicas a cumplir. Las condiciones adicionales (de carácter dimensional, funcional, etc) introducirán nuevas limitaciones, debiendo tratar de cumplir todas ellas empleando criterios heurísticos o de otro tipo.

\section{Posiciones relativas de los rectángulos}

Para estudiar las posibles posiciones relativas entre los distintos rectángulos, en primer lugar, se analizarán los vértices del grafo correspondientes a rectángulos externos, los cuales se ordenarán de forma consecutiva, formando un ciclo hamiltoniano.

Entre los rectángulos externos, pueden distinguirse tres clases:

Clase A.- Vértice externo de grado 2, vertical a otro. Ocupa necesariamente una sola esquina del rectángulo total, pues ha de tener dos rectángulos exteriores adyacentes cubriendo exactamente dos de sus lados contiguos. Su dimensión $I_{x}$ coincide con la del rectángulo adyacente verticalmente, y la $\left.\right|_{y}$ con la del adyacente horizontalmente (figura 13A).

Clase B.- Vértice externo de grado 2, no vertical a otros. Puede ocupar una o dos esquinas contiguas del rectángulo total. Los dos rectángulos adyacentes a él han de estar en contacto. Si ocupa una sola esquina, bien la dimensión $\left.\right|_{x}$ del adyacente verticalmente 0 la $\mathrm{l}_{\mathrm{y}}$ del adyacente horizontalmente han de coincidir con la correspondiente del de esquina, en tanto que la otra dimensión del otro rectángulo adyacente ha de ser mayor que la misma del de esquina (figura 13B y 13C). Si ocupase dos esquinas, los dos rectángulos adyacentes deben cubrir una dimensión completa (figura 13D).

Clase C.- Vértice externo de grado mayor que 2. Puede ocupar una o dos esquinas del rectángulo total, o una posición entre esquinas (figura 14).

Si en la capa externa hubiese dos vértices de la clase $\mathrm{A}$ consecutivos, entre ambos cubrirían un rectángulo con una dimensión total completa (figura 15A), por lo que se podría pasar a un grafo más sencillo, eliminando ambos vértices $\mathrm{y}$ obligando a que todos los vértices adyacentes a los eliminados cubran una dimensión completa. Sólo puede haber dos vértices de la clase $B$ juntos en el grafo $K 3$, pues ambos serían adyacentes a uno que exigiría una dimensión completa, por lo que habría dado lugar a subgrafos separables (figura 15B). Por otra parte, un vértice de la clase $\mathrm{A}$ no puede ser adyacente a uno de la clase $B$. En consecuencia, si existiesen vértices de las clases $A \circ B$, estarán flanqueados por vértices de la clase $C$.

Si dos rectángulos externos no consecutivos fuesen adyacentes, deberán estar en contacto con lados distintos del rectángulo total (figura 16).

Si un conjunto de rectángulos debe cubrir una dimensión completa, los extremos ocuparán al menos una esquina de dicho lado.

En general, existirán varias configuraciones de rectángulos externos cubriendo los cuatro lados del rectángulo total que respeten las reglas anteriores.

Podemos describir la situación de los rectángulos exteriores mediante la enumeración de los lados del rectángulo total con los que tiene contacto. Si numerásemos de forma consecutiva los lados del rectángulo total, llamando 1 al lado izquierdo y recorriésemos los otros lados en sentido horario, en el caso de la figura 11 , los rectángulos exteriores se describirían como: 1-2 el rectángulo 8, 2 el 9, 2-3 el $10,3-4$ el 3, 4 el 2 y $4-1$ el 1 .

Veamos cómo describir las posiciones de los rectángulos de la envoltura respecto al de base. Al considerar un rectángulo cualquiera, los rectángulos de su envoltura pueden tener contacto con él arriba, abajo, a la derecha, a la izquierda, o en una de sus cuatro esquinas. Si llamamos I, u, r y d a los lados izquierdo, arriba, derecho y abajo de un rectángulo, y con las dos letras correspondientes a las esquinas, podemos describir las posiciones relativas entre ellos. Vamos a considerar primero los cuadros externos y las conexiones entre ellos. En el ejemplo de la figura 11, tendremos: 

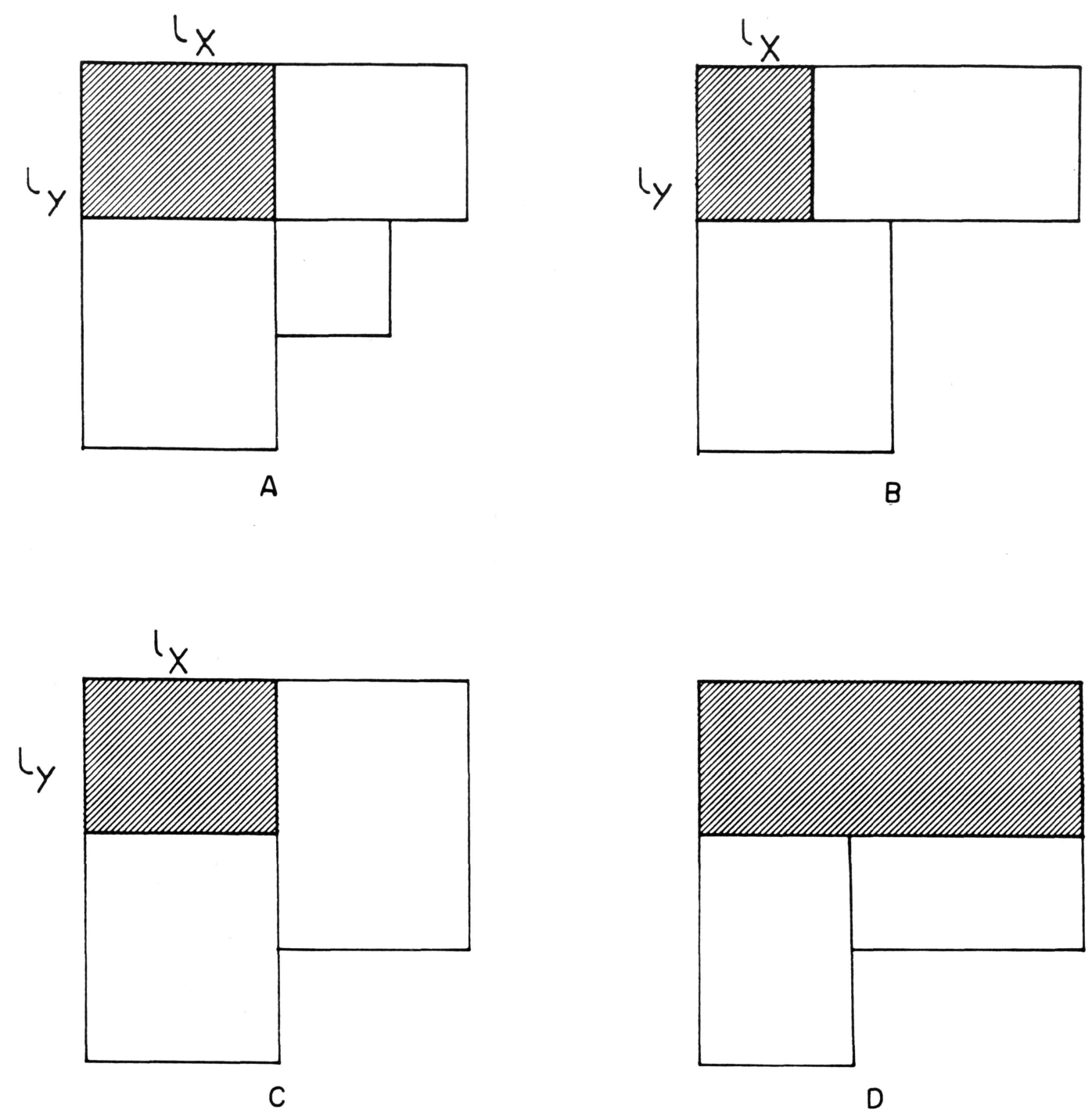

Fig. 13
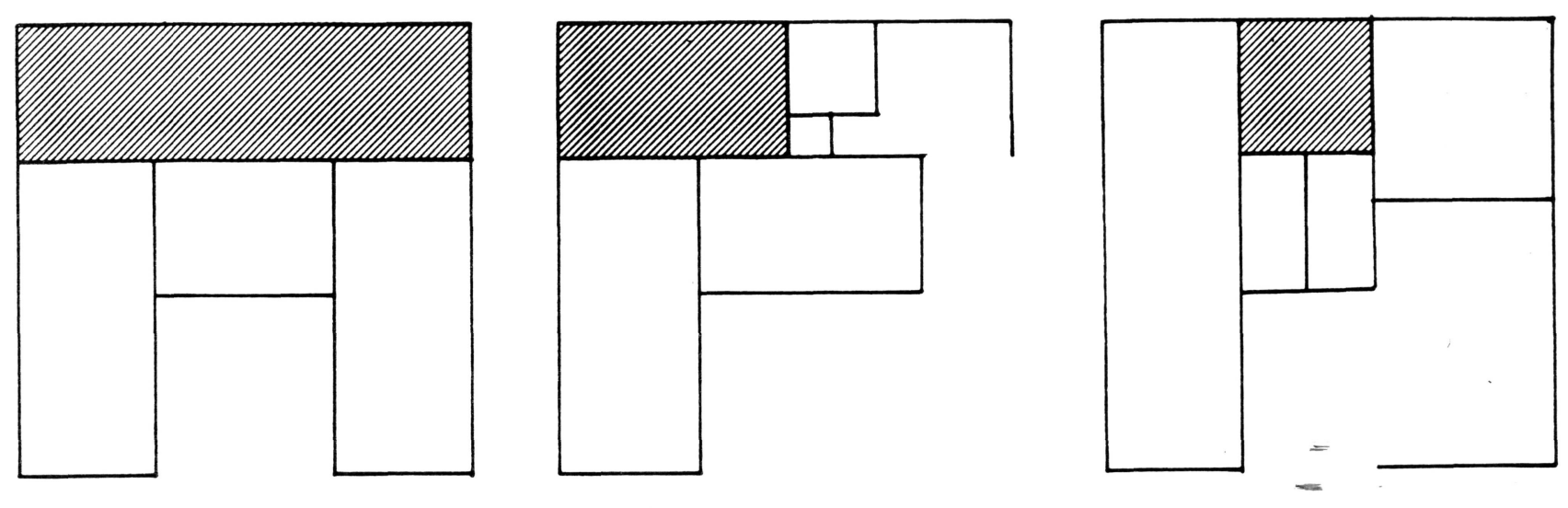

(c) Consejo Superior de Investigaciones Científicas

Fig. 14 Licencia Creative Commons 3.0 España (by-nc) 


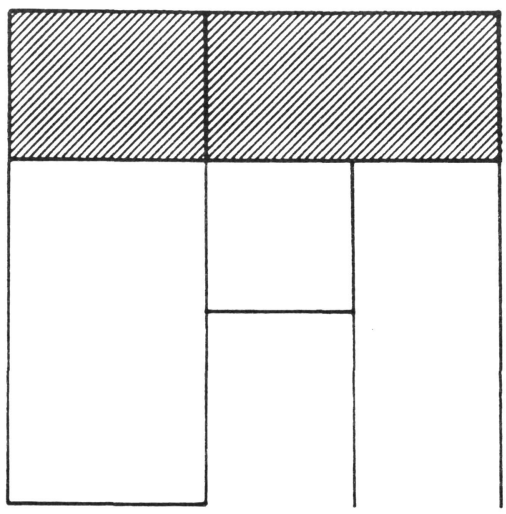

A

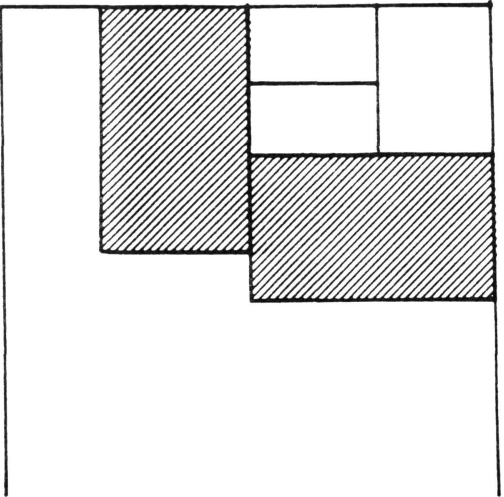

A

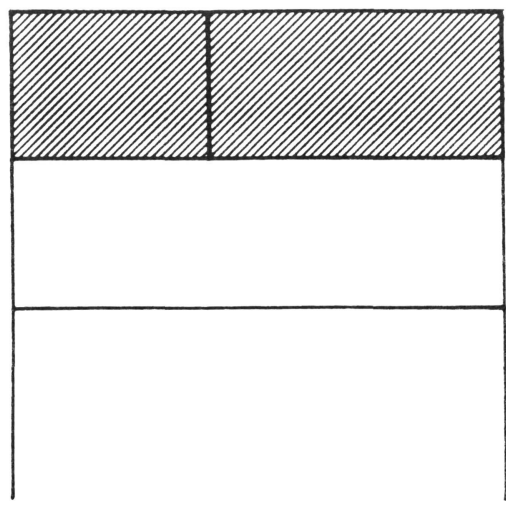

B

Fig. 15

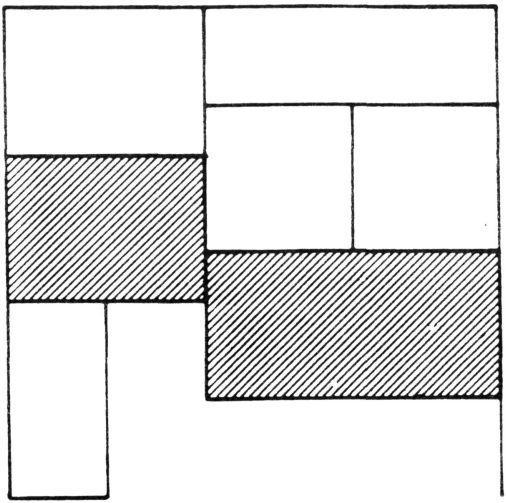

B rect 1 : u 8 ; ur $9 ;$ r 2 .

rect 2 : I 1 ; lu $1 ;$ ur $3 ;$ r 3 .

rect 3 : I 2 ; lu $10 ;$ u 10.

rect $10: 19 ; \mathrm{d} 3$.

rect 9: I 8; r 10; dl 1 .

rect $8: \mathrm{r} 9 ; \mathrm{d} 1$. rect $8: \operatorname{ld} 7$

rect 9: $\mathrm{d} 7$.

rect $7: I 1 ;$ lu $8 ;$ u $9 ;$ d $6 ;$ dl 1 .

rect $6:$ I $1 ;$ lu $1 ;$ u $7 ; \mathrm{d} 4 ;$ dl 1 .

rect $4: I 1 ;$ lu $1 ;$ u $6 ;$ d $2 ;$ dl 1 .

rect $2: \mathrm{u} 4$.
Seguidamente se analiza la envoltura de cada uno de los rectángulos externos, empezando a situar rectángulos internos. Al considerar la envoltura del rectángulo 1, los rectángulos 7, 6 y 4 deben estar a la derecha y colocados de arriba a abajo. Esto se deduce al considerar su orden en la envoltura, que 9 ocupa la esquina superior derecha (ur) y que 2 ocupa la derecha (r). Esto nos permite además fijar posiciones relativas entre los rectángulos de la envoltura. Así, añadiremos:
Este proceso se continúa tomando los vértices en el orden establecido anteriormente, añadiendo las nuevas informaciones relativas al rectángulo respecto a su envoltura y de los rectángulos de la envoltura entre sí.

\section{Generación de todas las configuraciones válidas de rectángulos externos}

A continuación se tratadegenerar todas las configuraciones 
válidas de rectángulos externos, sin incluir las simetrías ni rotaciones de la misma solución. A efectos de exposición, se introducen las siguientes variables, todas ellas vectores unidimensionales enteros de NE (número de vértices externos) elementos.

$\mathrm{LAB}(\mathrm{I})$, contiene los números de los vértices externos, ordenados en forma de ciclo hamiltoniano.

NMAX(I), NMIN(I), contienen respectivamente el número mínimo y máximo de esquinas del rectángulo total que puede tener el rectángulo I en una realización del grafo.

NACT(I), contiene las esquinas del rectángulo total que ocupa el rectángulo I en la realización en curso. La suma de todos los elementos de este vector ha de ser 4 .

En primer lugar, se debe asignar valores a NMIN y a NMAX. El procedimiento es como sigue:

Haciendo variar I desde 1 hasta NE:

Si $L A B(I)$ es de clase A: MIN(I) $=1 ; \operatorname{MAX}(I)=1$.

Si $\mathrm{LAB}(\mathrm{I})$ es de clase $\mathrm{B}$ : $\mathrm{MIN}(\mathrm{I})=1 ; \operatorname{MAX}(\mathrm{I})=2$.

Si LAB(I) es de clase C: $M I N(I)=0 ; \operatorname{MAX}(I)=2$.

El proceso consiste en generar todas las posibles combinaciones de los elementos de NACT, entre sus valores mínimo y máximo, de modo que su suma sea 4 .

Las verificaciones adicionales, tales como que varios rectángulos cubran una dimensión completa o que dos rectángulos estén en distintos lados del rectángulo total, deben hacerse sobre una propuesta de solución completa.

Así, en el ejemplo de la figura 1 , donde los vértices 1 y 3 son de la clase $B$, y el 2 y 4 de la clase $C$, las 8 soluciones que se obtienen se muestran en la figura 17. Los valores que toman los elementos de NACT y su traducción en lados del rectángulo total con los que tienen parte común son:

sol 1: $1,0,1,2 ; 1-2,2,2-3,3-4-1$

sol 2: $1,0,2,1 ; 1-2,2,2-3-4,4-1$

sol 3: $1,1,1,1 ; 1-2,2-3,3-4,4-1$

sol 4: $1,1,2,0 ; 1-2,2-3,3-4-1,1$

sol 5: $1,2,1,0 ; 1-2,2-3-4,4-1,1$

sol 6: $2,0,1,1 ; 1-2-3,3,3-4,4-1$

sol 7: $2,0,2,0 ; 1-2-3,3,3-4-1,1$

sol 8: $2,1,1,0 ; 1-2-3,3-4,4-1,1$

Todas las soluciones cumplen la condición adicional de que los rectángulos 2 y 4 , en contacto y no consecutivos, estén en distinto lado del rectángulo total. Si estableciésemos las permutaciones circulares de los cuatro lados del rectángulo total, es decir 1-2-3-4, 2-3-4-1, 3-4-1-2, y 4-1$2-3$, se multiplicaría por 4 el número de soluciones. $\mathrm{Si}$ además considerásemos las simétricas, es decir 4-3-2-1, 14-3-2, 2-1-4-3 y 3-2-1-4, multiplicaríamos por 2 el número total de soluciones, obteniendo un total de 64 realizaciones en un caso tan simple como el planteado.

En el ejemplo de la figura 11, el vértice 8 es de la clase $A$, y los $9,10,3,2$ y 1 son de la clase $C$. Si no hay condiciones adicionales, el algoritmo produce 30 soluciones, que se transforman en 240 si se tienen en cuenta las rotaciones y las simetrías.

\section{Elección de los grados de libertad de la partición}

La posición y dimensiones de un rectángulo quedan determinadas como se muestra en la figura 18 por las coordenadas de sus vértices inferior izquierdo $\left(\mathrm{x}_{0}, \mathrm{y}_{0}\right) \mathrm{y}$ superior derecho $\left(\mathrm{x}_{1}, \mathrm{y}_{1}\right)$.La situación relativa de los rectángulos adyacentes y verticales condiciona los valores de algunas de las coordenadas de referencia de los mismos. Consideremos los rectángulos situados en la parte superior y llamemos $\left(x^{\prime}{ }_{0}, y^{\prime}{ }_{0}, x^{\prime}, y_{1}\right)$ y $\left(x^{\prime \prime}, y_{0}{ }_{0}, x^{\prime \prime}, y_{1}{ }_{1}\right)$ a las coordenadas de los vértices extremos de dos consecutivos:

- En cualquier caso, $\mathrm{y}_{0}{ }_{0}=\mathrm{y}_{1} ; \mathrm{y}_{1}>\mathrm{y}_{0}$.

- Si es vertical por la esquina superior izquierda (figura $18 \mathrm{~A}), \mathrm{x}_{0}<\mathrm{x}_{0} ; \mathrm{x}_{1}{ }_{1}=\mathrm{x}_{0}$.

- Si ocupa la esquina izquierda y parte superior (figura 18B), $x_{0}^{\prime}<x_{0} ; x_{1}^{\prime}>x_{0}$.

- Si ocupa la parte superior, sin ocupar esquinas (figura $18 \mathrm{C}), x_{0}^{\prime} \geq x_{0} ; x_{1}^{\prime} \leq x_{1}$. Los signos de igualdad corresponden a los rectángulos más a la izquierda y más a la derecha, respectivamente.

- Si ocupa la parte superior y la esquina derecha (figura 18D), $x_{0}<x_{1} ; x_{1}^{\prime}>x_{1}$.

- Si ocupa la esquina derecha (figura $18 \mathrm{E}$ ), $x_{0}^{\prime}=x_{1}$; $x^{\prime}>x_{1}$.

- Si hubiese varios rectángulos superiores, entre cada dos consecutivos (figura $18 \mathrm{~F}$ ), $\mathrm{x}_{0}^{\prime \prime}=\mathrm{x}^{\prime}{ }_{1}$.

De forma análoga, pueden establecerse relaciones semejantes para los otros tres lados.

Veamos ahora, sobre el ejemplo de la figura 11, como la aplicación de estas reglas permite introducir los parámetros que representan los GDL de la partición. Si consideramos primero el vértice 1 y su envoltura, las coordenadas definitorias de los rectángulos quedarán

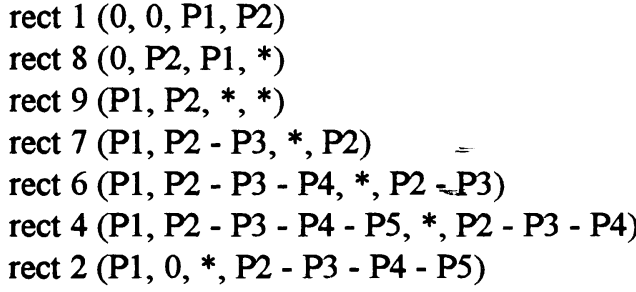



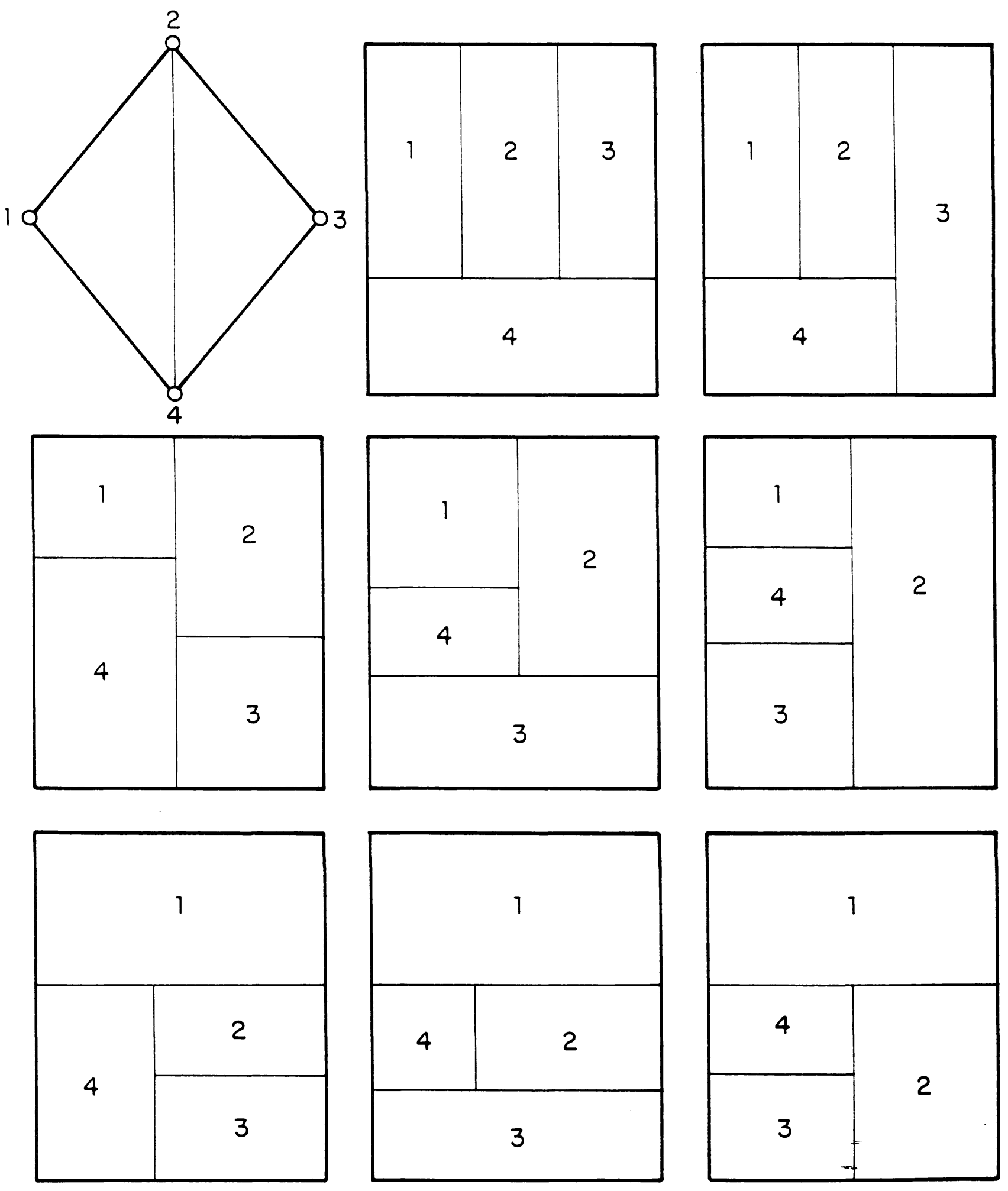

Fig. 17

(c) Consejo Superior de Investigaciones Científicas Licencia Creative Commons 3.0 España (by-nc) 


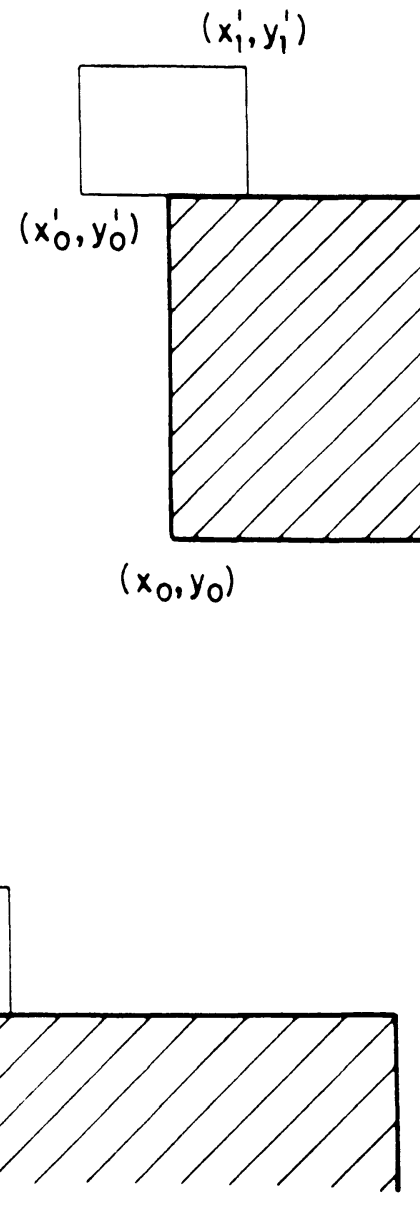

B

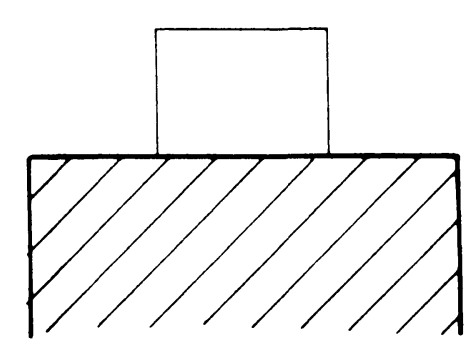

c

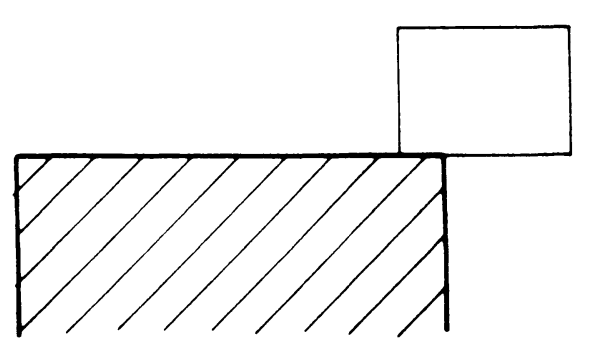

$\mathrm{D}$

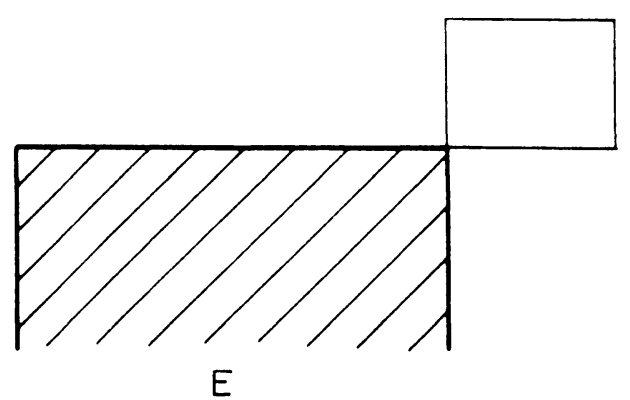

$\mathrm{E}$

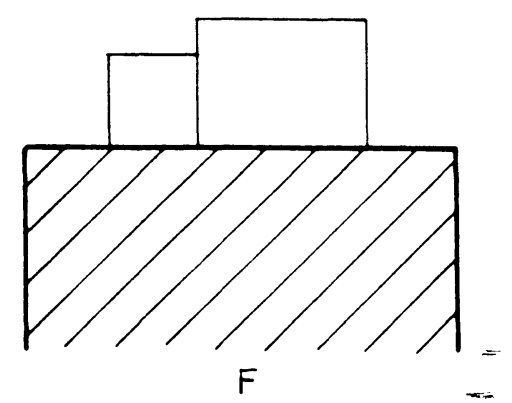

Fig. 18 
Al considerar el 8 y su envoltura, las modificaciones son:

$$
\text { rect } 8(0, \mathrm{P} 2, \mathrm{P} 1,1)
$$$$
\text { rect } 9(\mathrm{P} 1, \mathrm{P} 2, *, 1)
$$

Al considerar el 9 y su envoltura, las modificaciones que introduce son:

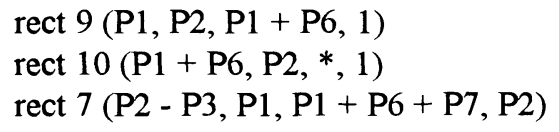

Al considerar el 10 y su envoltura, las modificaciones serán:

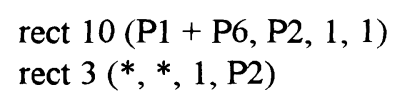

Al considerar el 3 y su envoltura, las modificaciones serán:

$$
\begin{aligned}
& \text { rect } 3(\mathrm{P} 1+\mathrm{P} 6+\mathrm{P} 7,0,1, \mathrm{P} 2) \\
& \text { rect } 2(\mathrm{P} 1,0, \mathrm{P} 2-\mathrm{P} 3-\mathrm{P} 4-\mathrm{P} 5, \mathrm{P} 1+\mathrm{P} 6+\mathrm{P} 7)
\end{aligned}
$$

rect 5 (*, P2 - P3 - P4 - P5, P1 + P6 + P7, P2 - P3 - P4) rect $6(\mathrm{P} 1, \mathrm{P} 2-\mathrm{P} 3-\mathrm{P} 4, \mathrm{P} 1+\mathrm{P} 6+\mathrm{P} 7, \mathrm{P} 2-\mathrm{P} 3)$

Al considerar el 2 y su envoltura, las modificaciones serán:

rect $4(\mathrm{P} 1, \mathrm{P} 2$ - P3 - P4 - P5, P1 + P8, P2 - P3 - P4)

rect $5(\mathrm{P}+\mathrm{P} 8, \mathrm{P} 2-\mathrm{P} 3-\mathrm{P} 4-\mathrm{P} 5, \mathrm{P} 1+\mathrm{P} 6+\mathrm{P} 7, \mathrm{P} 2-\mathrm{P} 3-\mathrm{P} 4)$

Donde se indica con * las coordenadas aún no fijadas, pudiendo ver en la figura 19 el significado geométrico de los parámetros $\mathrm{P} 1, \mathrm{P} 2, \ldots$. . Como ya se ha indicado, este conjunto de parámetros depende de los criterios que se adopten en su elección, aunque el número de parámetros independientes es conocido. Los condicionantes dimensionales establecerán relaciones entre estos parámetros, en forma de ecuaciones o inecuaciones. Si este sistema fuese incompatible, la realización sería válida topológicamente, pero no geométricamente.

\section{Aplicación a plantas no convexas}

El método puede ser utilizado con plantas no convexas, cuando todos sus lados sean paralelos a dos direcciones perpendiculares. En tal caso, el rectángulo total será el rectángulo envolvente mínimo. Deberán añadirse uno o varios rectángulos, hasta completar dicho rectángulo envolvente, a los que se asignarán los vértices y aristas correspondientes en el grafo a realizar. En las realizaciones que se propongan, los rectángulos correspondientes a estos vértices adicionales deberán ocupar posiciones prefijadas, $y$, posiblemente, tener dimensiones fijas.

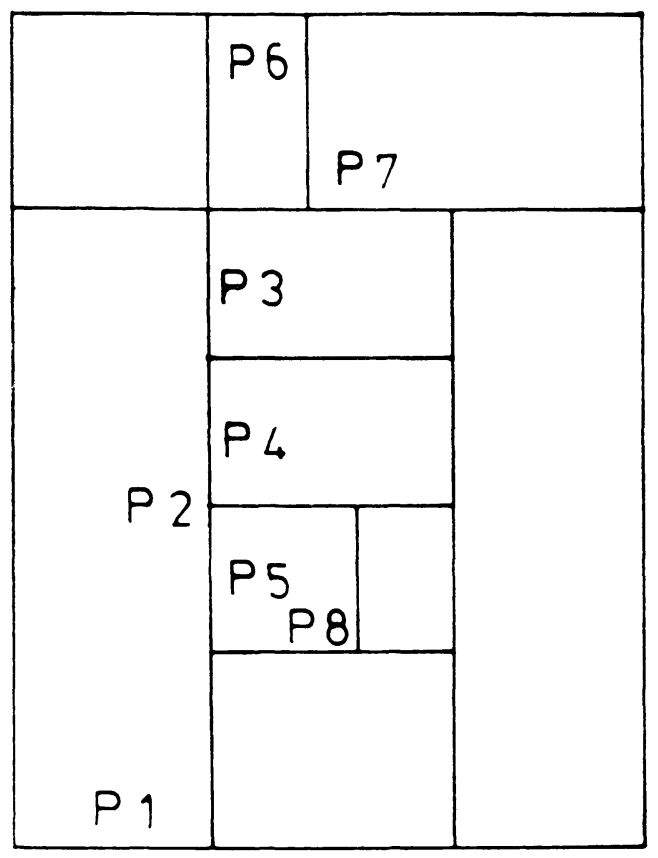

Fig. 19

En la figura 20 se muestran dos ejemplos de plantas no convexas, y sendos grupos de rectángulos adicionales, en trazo discontinuo, que completan el rectángulo envolvente mínimo.

\section{1.- Paso de una planta cualquiera a una rectangular}

Se denominará planta básica a la planta a cubrir y planta ampliada al rectángulo envolvente mínimo. Se denominará planta adicional a la diferencia entre las plantas ampliada y básica.

La planta básica puede caracterizarse por el número máximo de extremos independientes que contiene. Se denominará así al máximo número de rectángulos que pueden estar en contacto con tres lados consecutivos de la planta básica. En el ejemplo de la figura 20A hay tres extremos independientes, y en el de la figura 20B hay 4 . Veamos una forma de describir la planta básica que sea compacta y de la que se puedan deducir fácilmente sus características.

Supóngase establecido un sentido de recorrido de los lados de la planta básica tal que en los lados externos, coincidentes con los de la planta ampliada, dicho sentido sea el horario. Los vértices se anotarán como 1 ó -1 según produzcan un giro a derechas o a izquierdas. En las proximidades de un vértice de tipo -1 , los dos lados pertenecerán a un mismo rectángulo, en tanto que en las de un vértice de tipo - 1 pertenecerán a dos rectángulos distintos. 


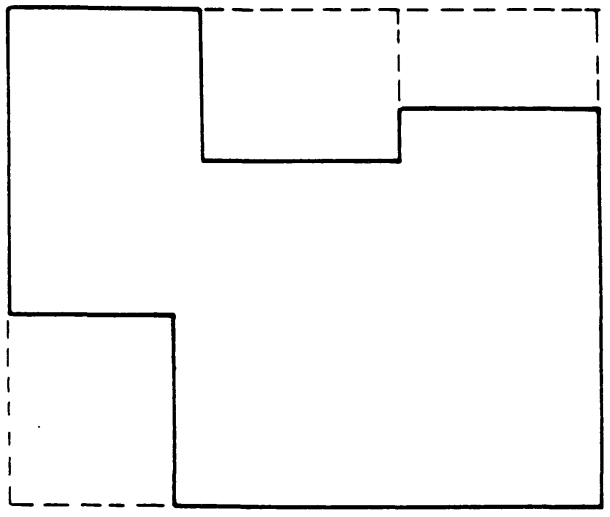

A

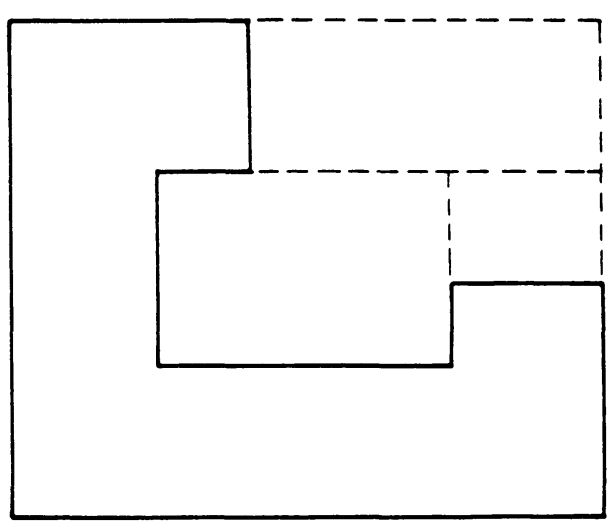

B
Esta secuenciacíclica de $1 \mathrm{y}-1$ proporcionauna descripción topológica de la planta básica. Tomando como origen un punto infinitamente próximo al comienzo de un lado horizontal y con sentido a la derecha, por ejemplo, y agregando a cada lado su longitud, se obtendrá una descripción geométrica de la planta básica. La secuencia de giros a derecha o a izquierda permite también calcular el número de extremos independientes de la planta básica.

La planta adicional puede descomponerse, a criterio del proyectista, en un conjunto de rectángulos de posición y dimensiones conocidas. Siempre es posible conseguir que parte de estos rectángulos cubran la parte de los lados de la planta ampliada no cubierta por lados de la planta básica, de modo que sean adyacentes a la planta básica. En la mayor parte de los casos, la planta adicional podrá ser cubierta por dichos rectángulos, pero en algunos casos, como en la figura $20 \mathrm{~B}$, serán necesarios rectángulos interiores a la planta ampliada que siempre podrán ser adyacentes a más de un lado de la planta básica.

La planta adicional está dividida en rectángulos de una forma determinada. Para describirla, de forma coherente con el procedimiento que se presenta para generar realizaciones, puede utilizarse la nomenclatura propuesta, tanto para describir su posición en el rectángulo ampliado como para describir sus posiciones relativas. Así, en el caso de la planta de la figura $20 \mathrm{~A}$, la planta adicional tiene dos partes disjuntas, formadas por uno y por dos rectángulos respectivamente. El rectánguloaislado ocupa la posición $4-1$, y los otros dos unidos las posiciones 1 y la $1-2$, respectivamente. De éstos, el primero ocupa las posiciones br y $r$ del segundo y, en consecuencia, éste ocupa parte de la posición 1 del primero.

\section{2.- Modificación del grafo a realizar}

Se denominará grafo básico a aquél cuyas realizaciones sobre la planta básica se desean obtener y grafo adicional al correspondiente a la disposición de rectángulos elegida para cubrir la planta adicional. El grafo adicional puede ser no conexo. Sus vértices pueden clasificarse en exteriores e interiores, respecto a la planta ampliada. Por lo que se refiere al grafo básico, al hacer el análisis de la envoltura de sus vértices, no son de aplicación todas las condiciones indicadas para el caso de planta básica rectangular.

El incumplimiento de alguna de las verificaciones indicará que el grafo básico no es realizable sobre la planta básica, pero el cumplimiento de todas no garantiza su realizabilidad. Hay que señalar que pueden existir realizaciones en las que se presenten rectángulos verticales entre sí, además de los que deben serlo obligatoriamente.

Los vértices del grafo básico seguirán denominándose exteriores o interiores, según que su envoltura sea abierta o cerrada. Los rectángulos correspondientes a vértices exteriores serán adyacentes a lados de la planta básica.

Es posible establecer un conjunto de conexiones admisibles u obligatorias entre vértices del grafo básico y vértices del grafo adicional. Así, si un vértice del grafo adicional representa un patio de manzana, puede establecerse que la cocina o un baño den a él, o que algún dormitorio secundario pueda dar a él. Puede entonces generarse un conjunto de grafos, que se denominarán grafos ampliados, formados por los grafos básico y adicional conectados mediante los vínculos obligatorios y las distintas combinaciones de vínculos admisibles.

La configuración concreta de cada planta adicional permite establecer reglas limitativas entre los vínculos entre vértices de los grafos básico y adicional. Así, si se considera el caso de la figura 20A, en la que la ptanta adicional está formada por tres rectángulos descritošen el sub-apartado anterior, paragenerar los grafos ampliados, los vértices del grafo adicional correspondientes a estos tres rectángulos 
deberán estar unidos con al menos 2,3 y 1 vértices del grafo básico, respectivamente.

Los dos últimos han de estar unidos a un vértice, y solo uno, común del grafo básico. El vértice aislado puede estar conectado con un vértice del grafo básico con el que esté conectado el primero de los otros dos, pero no puede estar conectado con ningún vértice del grafo básico conectado con el segundo.

Sin embargo, ciertas reglas son de aplicación general, cualquiera que sea la planta considerada. Así, se tendrá que:

- Sólo se establecerán nuevos vínculos entre los vértices exteriores del grafo básico y vértices del grafo adicional.

- Todos los vértices exteriores del grafo adicional se conectarán con algún vértice del básico.

- Todos los vértices interiores del grafo adicional se conectarán al menos con dos vértices del básico.

- Dos vértices externos del grafo adicional adyacentes sólo pueden estar conectados a un vértice común del grafo básico.

- Un vértice aislado del grafo adicional ha de estar conectado a dos o más vértices del grafo básico.

Los grafos ampliados han de ser realizados sobre una planta rectangular, por lo que les es aplicable el procedimiento descrito para saber si son realizables, y en tal caso, generar realizaciones de los mismos.

\section{Conclusiones}

El problema de saber si un grafo es realizable en recintos rectangulares que cubran una planta dada de forma cualquiera, de lados paralelos a dos direcciones perpendiculares, no parece abordable de forma directa. La vía elegida por los autores ha sido la de considerar, en primer lugar, el caso de planta rectangular. Resuelto el problema en dicho caso, se propone una forma de reducir el caso general al caso resuelto, mediante la introducción de grafos auxiliares, que complican el grafo de partida, pero que permiten pasar de un problema no resuelto a otro resuelto.
El problema de determinar si un grafo admite realizaciones en rectángulos que cubran otro rectángulo es de interés práctico en áreas tales como el proyecto arquitectónico o la compactación de circuitos electrónicos, entre otras. Los autores no han encontrado referencias a una solución general del mismo, en la bibliografia consultada. En este trabajo se consideran los dos aspectos del problema: saber si el grafo admite este tipo de realizaciones; y, en caso afirmativo, generar automáticamente realizaciones del mismo.

Respecto al primero de ellos, se presenta un método que hace uso de un conjunto de condiciones necesarias, implementado mediante un algoritmo que permite determinar si un grafo puede considerarse realizable y que está basado fundamentalmente en el análisis de la envoltura de cada vértice. El grado de complejidad del algoritmo es el número de vértices al cuadrado.

En el caso en que el grafo fuese realizable, se analizan las condiciones que han de cumplir los rectángulos externos, de forma que puedan generarse disposiciones válidas de los mismos, que servirán de base a realizaciones del grafo. Se presenta un algoritmoque permite generar automáticamente todas las configuraciones válidas que pueden tomar los rectángulos externos.

Se presenta un método que permite generar realizaciones del grafo, a partir de una distribución de los rectángulos externos. En dicha realización, se introducen los parámetros que representan sus grados de libertad, cuyo valor ha de fijarse atendiendo a condiciones adicionales, de tipo geométrico, organizativo funcional, etc, utilizando procedimientos heurísticos, de programación lineal, u otros, según el problema de que se trate.

Por último, se indica la forma de aplicar el procedimiento propuesto a plantas no convexas, siempre que sus lados sean paralelos a dos direcciones perpendiculares. Se muestra como pasar de la planta no convexa a la convexa, y de modo correspondiente, del grafo dado a un conjunto de grafos ampliados que deben realizarse sobre la planta convexa.

\section{REFERENCIAS}

P. UNGAR: “On diagrams representing maps", J. London Math. Soc., vol 28, 1953 pp $336-342$.

P. H. LEVIN: "Use of graphs to decide optimum layout of buildings", Archit. J., 1966, pp 809 - 817.

W. MITCHELL, J.P. STEADMANy R. S. LIGGETT: "Synthesis and optimisation of small rectangular floor plans", Environment and planning, vol. 3, 1976, pp 37 - 70.

C. H. EARL y L. J. MARCH: “Architectural applications of graph theory", en Applications of graph theory" edit. por Wilson y Beinek, Academic Press, Londres, 1979, pp 327 - 355.

J. MAGIERA: "Exterior partitions of a rectangle into rectangles and their graphs", en "Graph Theory, Lagow 1981 proceedings" 
edit. por Borowiecki et al., Springer Verlag, Berlin 1981pp 125 -130 .

J. ROTH R., HASHIMSHONY y A. WACHMAN: "Turning a graph into a rectangular floor plan", Building \& Environment, vol 17, 1982, pp 163 - 173. J.

P. STEADMAN: “Architectural morphology”, Pion Itd., Londres 1983.

J. ROTH y R. HASHIMSHONY: "Algorithms in graph theory and their use for solve problemms in Architectural design", Computer aided design, vol 20, 1988, pp $373-381$.

A. SCHWARZ, D. M. BERRY y E. SHABIB: "Representing and solving the automatic building design problem", Computer Aided Design, vol 26.9, 1994, pp 689 - 698.

A. SCHWARZ, D. M. BERRY y E. SHABIB: "The use of automatic building design system", Computer Aided Design, vol $26.10,1994$, pp $747-761$.

A. RECUERO: "Aplicaciones de la teoría de grafos: Búsqueda de caminos en una red y análisis de su conectividad", Informes de la Construcción, vol. 46, nº 433, 1994, pp 33 - 45.

A. RECUERO y O. RÍO: "Aplicación de la teoría de grafos a la planificación y programación de proyectos", Informes de la Construcción, vol. 46, $\mathrm{n}^{\circ} 431,1994$, pp 49 - 60.

A. RECUERO, M. ALVAREZ y O. RÍO: "Grafos en el proyecto arquitectónico: Partición de un rectángulo en rectángulos", Métodos numéricos para el cálculo y diseño en Ingeniería, (en prensa).

A. RECUERO, M. AlVAREZ, y O. RÍO: "Partition of a rectangle into rectangles, and its application to a architectural design". 2nd Workshop of the European Group for Structural Engineering Applications of Artificial Intelligence (EG-SEAAI). Bergamo, Italy, 1995.

\section{publicación del IETCC/CSIC}

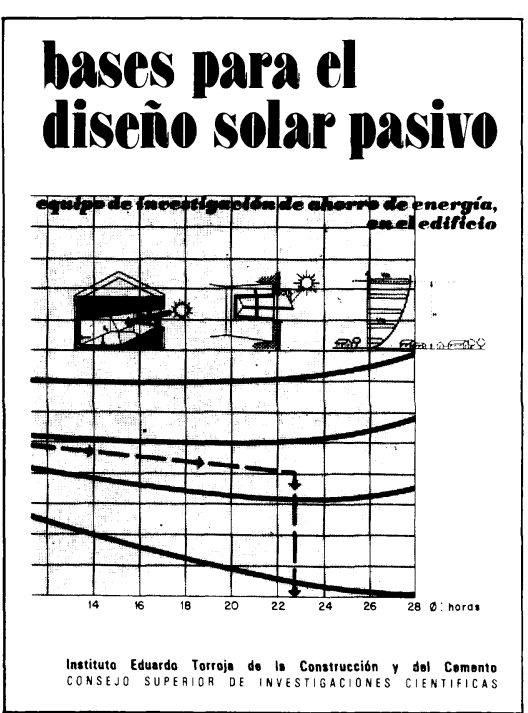

Equipo de Ahorro de Energia en el edificio

Dirección y coordinación: Arturo García Arroyo

M. ${ }^{a}$ José Escorihuela José Luis Esteban José Miguel Frutos Manuel Olaya Bernardo Torroja
Las dificultades de suministro $y$ el alto coste de los productos energéticos convencionales han despertado la atención de los usuarios, técnicos e industriales de la edificación hacia los procedimientos y sistemas en que se basa el aprovechamiento de otras fuentes alternaha generado un rápido desarrollo industrial y comercial que, en opinión de los autores de este libro, arrastran los siguientes defectos: un mimético tecnologismo respecto de los sistemas convencionales que violenta las peculiaridades de la energía solar (baja densidad y variabilidad en el tiempo), y una escasa selectividad en la aplicación de los sistemas y procedimientos pasivos dando origen a un ecumenismo arquitectónico solar, al margen de las condiciones climáticas y funcionales específicas de cada caso y lugar.

En este libro, utilizando criterios y metodologia pedagógicos, se dan los fundamentos e instrumentos té́rico-prácticos necesarios para el planteamiento de todo proyecto arquitectónico solar pasivo, de acuerdo con los principios éticos y económicos de conservación y ahorro de energía. Es decir: respeto de los presupuestos bioclimáticos, búsqueda de la máxima captación y acumulación de la radiación solar, y esmero en el aislamiento térmico de los cerramientos.

Un volumen encuadernado en cartulina ibiza plastificada, a cinco colores, de $16 \overline{\times} 23 \mathrm{~cm}$, compuesto de 216 páginas, 217 figuras, 87 gráficos, 19 tablas y 10 cuadros. 